Math. Model. Nat. Phenom.

Vol. 7, No. 1, 2012, pp. 166-202

DOI: $10.1051 / \mathrm{mmnp} / 20127199$

\title{
Mathematical Modeling of Leukemogenesis and Cancer Stem Cell Dynamics
}

\author{
T. Stiehl and A. Marciniak-Czochra * \\ Interdisciplinary Center for Scientific Computing (IWR) \\ Institute of Applied Mathematics and BIOQUANT \\ University of Heidelberg, Im Neuenheimer Feld 267 \\ D - 69120 Heidelberg, Germany
}

\begin{abstract}
The cancer stem cell hypothesis has evolved to one of the most important paradigms in biomedical research. During recent years evidence has been accumulating for the existence of stem cell-like populations in different cancers, especially in leukemias. In the current work we propose a mathematical model of cancer stem cell dynamics in leukemias. We apply the model to compare cellular properties of leukemic stem cells to those of their benign counterparts. Using linear stability analysis we derive conditions necessary and sufficient for expansion of malignant cell clones, based on fundamental cellular properties. This approach reveals different scenarios of cancer initiation and provides qualitative hints to possible treatment strategies.
\end{abstract}

Key words: multi-compartmental models, stem cell differentiation, environmental signaling, leukemia, cancer stem cells, leukemic stem cells, linear stability analysis

AMS subject classification: 34D20, 34D35, 92D25, 92C50, 92C37

\section{Introduction}

Tissues of complex organisms consist of different types of specialized cells. In many tissues a small population of unspecialized cells, so called adult stem cells (tissue stem cells) gives rise to all cell types of the tissue. Adult stem cells are characterized by the potential to produce different cell types and the ability to maintain the size of their population by a process referred to as

*Corresponding author. E-mail: anna.marciniak@iwr.uni-heidelberg.de 
self-renewal. Adult stem cells ensure tissue maintenance and regeneration [56, 51]. Formation of mature specialized cells is a multi-step process starting from stem cells that give rise to a hierarchy of immature progenitor cells which develop into mature cells. The hematopoietic (blood-forming) system with hematopoietic stem cells at the top of the hierarchy is a known example of a multistage system.

During last years the concept of tissue stem cells has been extended to the concept of cancer stem cells (cancer initiating cells). According to this hypothesis a small population of "stem-like" cancer cells gives rise to the bulk of malignant cells $[18,20]$. Since cancer stem cells may be difficult to access by classical treatment strategies, they are thought to be responsible for relapse of malignancies $[17,63]$. Cancer stem cells have already been described in the context of different cancers $[18,15,33]$.

Because of relative facility of sampling blood or bone marrow, the hematopoietic system is well suited for modeling and validation. Hematopoiesis involves complex processes that can be examined at the level of genes, signal transduction proteins, or the populations of diverse cell types. By viewing hematopoiesis as a dynamical system and stress and disease as a perturbation of the system, the system biology approach may contribute to a better understanding of physiological and pathological states of hematopoiesis such as leukemias.

Depending on specific scientific questions, different mathematical models were developed to study hematopoiesis. One established method of modeling of multistage cell systems is to use a discrete collection of ordinary differential equations, each of which describes dynamics of cells at a single maturation stage $[8,22,39,43,47,64,50]$. Describing cell differentiation as a continuous process leads to models based on partial differential equations of the transport type [3, 11, 25, 24] or delay differential equations [27, 44, 4, 2, 55, 6]. Another group of models addresses the effects of stochasticity in the cell fate decisions $[65,66]$ or in the regulation of cell quiescence [59]. These models were designed to simulate the time dynamics of blood reconstitution, spread of cancer stem cells or perturbations in the blood dynamics in diseases such as e.g., myeloid leukemias, and provided explanations for a range of experimental and clinical observations. Whenever cell numbers are small, stochasticity plays an important role. This is, e.g., the case at the outset of cancer or before complete extinction of malignant clones. Reference [23] discusses a mathematical model for these scenarios, based on the Moran process taking into account differences in the reproductive fitness of different subpopulations. As proposed in ref. [32], stochastic models allow localizing the origin of leukemia stem cells in the cellular hierarchy of the hematopoietic system at the level of progenitor cells. So called agent-based models allow considering cellular heterogeneity and environmental interactions at the single-cell level. Fitted to experimental data, they can provide insights into clinical scenarios such as imatinib treatment [58]. Enormous computational costs for systems with large cell numbers make it necessary to approximate them by continuous partial differential equation (PDE) (or ordinary differential equation; ODE) models [57].

In the current work we investigate how changes in parameters describing proliferation rates and 
self-renewal properties may influence the dynamics of healthy and leukemic cell populations. For this task we propose a mathematical model that is an extension of our models of healthy hematopoiesis. Although the model focuses on the biology of the hematopoietic system, it may be relevant for other cellular systems, since different stem cell systems share common traits [56, 51].

The paper is organized as follows: In Section 2. we introduce a model of leukemia and compare it to the model of healthy hematopoiesis proposed in ref. [47] and analyzed in ref. [64, 30, 54]. In Section 3. we investigate the existence of multiple steady states and analyze which cell properties are necessary and sufficient to destabilize the physiological equilibrium of healthy cells. We interpret the mathematical results and conclude in which respect the dynamics of cancer stem cells may differ from the dynamics of tissue stem cells and healthy progenitors. Based on the mathematical results we discuss different scenarios of cancer initiation and give examples of hematologic malignancies which may follow these scenarios. We perform linear stability analysis of the system consisting of stem cells, mature cells, leukemic stem cells and a population of non-stem leukemic cells. In Section 4. we present numerical solutions illustrating the results of the analysis. We conclude in Section 5. with summary and final comments. Technical proofs are deferred to the Appendix.

\section{Model}

In the following we develop a mathematical model of time dynamics of leukemic cell populations. The model is an extension of our models of healthy hematopoiesis [47, 48, 64]. Based on the classical understanding of hematopoiesis we assume that the hematopoietic system consists of an ordered sequence of discrete maturation states, so called compartments, which are sequentially traversed [34]. We treat each compartment as a "well-mixed tank" and describe time evolution using ordinary differential equations. The enormous amount of cells forming the hematopoietic system $[34,41]$, justifies this approach. The model includes one leukemic cell lineage and one healthy cell lineage. We assume that the healthy hematopoietic cell line consists of $n$ compartments, where the first compartment is the population of hematopoietic stem cells (HSC) and the $n$th compartment is the population of post-mitotic mature cells, e.g., mature white blood cells. Similarly, we assume that the leukemic cell line consists of $m$ compartments, where leukemia initiating cells (LIC, "leukemic stem cells") are the first compartment. We denote by $c_{i}(t)(i=1, \ldots, n)$ the cell densities in the compartment $i$ of the healthy hematopoiesis at time $t$ and by $l_{i}(t)(i=1, \ldots, m)$ the corresponding cell densities in the leukemic lineage.

Healthy cells in compartment $i$ are characterized by the following properties.

- $p_{i}^{c}(t)$ : Proliferation rate in compartment $i$ at time $t$, describing how often a cell divides per unit of time. We assume that cells of the most mature compartment are post-mitotic, i.e. $p_{n}^{c}(t) \equiv 0$.

- $a_{i}^{c}(t)$ : Fraction of self-renewal in compartment $i$ at time $t$, i.e., the fraction of daughter cells returning to the compartment occupied by the mother cells that gave rise to them. Based on 
our earlier work [47] we assume that the fraction of self-renewal is regulated by feedbacksignaling.

- $d_{i}^{c}$ : Death rate in compartment $i$, describing which fraction of cells dies per unit of time. For simplicity, death rates are assumed to be zero or constant in time.

Corresponding parameters of the leukemic cell line are denoted by $p_{i}^{l}(t), a_{i}^{l}(t)$ and $d_{i}^{l}$. We assume $p_{m}^{l}(t) \equiv 0$.

\section{Healthy Hematopoiesis}

\section{Population Dynamics}

Based on the notation specified above, the flux to mitosis in compartment $i<n$ at time $t$ equals $p_{i}^{c}(t) c_{i}(t)$. During mitosis the mother cell disappears and gives rise to two daughter cells. The outflux from mitosis at time $t$ equals $2 p_{i}^{c}(t) c_{i}(t)$, of which the fraction $2 a_{i}^{c}(t) p_{i}^{c}(t) c_{i}(t)$ stays in compartment $i$ (process referred to as self-renewal). The fraction $2\left(1-a_{i}^{c}(t)\right) p_{i}^{c}(t) c_{i}(t)$ moves to compartment $i+1$ (process referred to as differentiation). Since stem cells are the most primitive cells, there exists no influx to the stem cell compartment due to differentiation. With the additional assumption that the most mature cells are post-mitotic, time evolution of the healthy hematopoietic compartments is described by the following system of equations,

$$
\begin{aligned}
\frac{d}{d t} c_{1}(t) & =\left(2 a_{1}^{c}(t)-1\right) p_{1}^{c}(t) c_{1}(t)-d_{1}^{c} c_{1}(t) \\
\frac{d}{d t} c_{i}(t) & =2\left(1-a_{i-1}^{c}(t)\right) p_{i-1}^{c}(t) c_{i-1}(t)+\left(2 a_{i}^{c}(t)-1\right) p_{i}^{c}(t) c_{i}(t)-d_{i}^{c} c_{i}(t), \quad 1<i<n, \\
\frac{d}{d t} c_{n}(t) & =2\left(1-a_{n-1}^{c}(t)\right) p_{n-1}^{c}(t) c_{n-1}(t)-d_{n}^{c} c_{n}(t) .
\end{aligned}
$$

\section{Signal Feedback}

Formation of healthy blood cells is regulated by a negative feedback $[42,49,28]$. If there is a need for more blood cells of a certain type, the concentration of signaling molecules, so called cytokines, increases and stimulates formation of mature cells. It is accepted that the rate of expansion of progenitor cells increases, if cytokine concentrations increase, either by enhanced proliferation or by reduced apoptosis $[28,34,5]$. Important examples of hematopoietic cytokines are erythropoietin (EPO) for erythropoiesis (red blood cell formation) and granulocyte colony stimulating factor (G-CSF) for granulopoiesis (formation of granulocytes, one type of white blood cells). However, the precise nature of this regulation is still unknown. As concluded from our work on healthy hematopoiesis $[47,64,46]$, the positive equilibrium can be stable in the models with a single nonlinear feedback inhibition of self-renewal fractions or of division rates. In the two-compartment model, the positive equilibrium is always stable. On the other hand, in the three-compartment model, emergence of an instability region in a parameter plane is possible [54]. These stability results were extended to a global stability in case of the two-compartment model 
[30]. Moreover, numerical solutions of the proposed model, validated based on the clinical observations of hematopoiesis after bone marrow transplantation $[47,46]$, indicate that the regulation of self-renewal is a more efficient mechanism than the regulation of proliferation rates. Similar conclusions were drawn using the models of multistage cell lineages applied to regeneration and maintenance of the mouse olfactory epithelium [39, 43].

Therefore, in the remainder of this paper we assume that the regulatory mechanism is based on the feedback inhibition of self-renewal depending on the level of mature cells. For simplicity we take only one cytokine into account. Based on knowledge of G-CSF [49, 42, 62], we assume that cytokine molecules are produced at a constant rate $\alpha$, degraded at a constant rate $\beta$ and eliminated proportionally to the density of mature cells at a constant positive rate $\mu$. Following [47], we obtain a time evolution of cytokine concentration given by

$$
\frac{d}{d t} S(t)=\alpha-\beta S(t)-\mu S c_{n}(t)
$$

where cytokine concentration at time $t$ is denoted by $S(t)$. Since cytokine dynamics is fast in comparison to cell cycle duration, $[14,52]$, we apply a quasi-steady state approximation. Substitution of $s(t):=\frac{\mu}{\alpha} S(t)$ and $k:=\frac{\beta}{\mu}$ leads to

$$
s(t)=\frac{1}{1+k c_{n}(t)} \in(0,1] .
$$

Since the exact relationship of cell properties and signal concentration is not known, we parsimoniously assume it to be linear, i.e. $a_{i}^{c}(t):=a_{i, \max }^{c} s(t)$, where $a_{i, \max }^{c} \in(0,1]$.

In the present work we focus on the impact of self-renewal on time dynamics of leukemia and assume proliferation rates to be constant in time. This results in the following model of healthy hematopoiesis,

$$
\begin{aligned}
\frac{d}{d t} c_{1}(t) & =\left(2 a_{1, \max }^{c} s(t)-1\right) p_{1}^{c} c_{1}(t)-d_{1}^{c} c_{1}(t), \\
\frac{d}{d t} c_{i}(t) & =2\left(1-a_{i-1, \max }^{c} s(t)\right) p_{i-1}^{c} c_{i-1}(t)+\left(2 a_{i, \max }^{c} s(t)-1\right) p_{i}^{c}(t) c_{i}(t)-d_{i}^{c} c_{i}(t), \quad 1<i<n \\
\frac{d}{d t} c_{n}(t) & =2\left(1-a_{n-1, \max }^{c} s(t)\right) p_{n-1}^{c} c_{n-1}(t)-d_{n}^{c} c_{n}(t), \\
s(t) & =\frac{1}{1+k c_{n}(t)}
\end{aligned}
$$

\section{Leukemic Cell Line}

There is evidence that the leukemic cell population consists of an ordered sequence of cell states, similar to the healthy hematopoietic cell lines $[15,33]$. The so-called leukemic stem cell (leukemia 
initiating cell, LIC) is the most primitive leukemic cell and gives rise to all leukemic cell types.

In the present model we assume that leukemic cells are stimulated by the same cytokines as healthy HSC. In other words, we assume that healthy cells and leukemic cells compete for environmental resources, being the hematopoietic cytokine $s(t)$. To model this competition we assume degradation of $s(t)$ by leukemic cells. Based on the steady state assumption we obtain:

$$
s(t):=\frac{1}{1+k^{c} c_{n}(t)+k^{l} l_{m}(t)},
$$

if we neglect signal degradation by primitive leukemic cell types, or otherwise

$$
s(t):=\frac{1}{1+k^{c} c_{n}(t)+\sum_{i=1}^{m} k_{i}^{l} l_{i}(t)}
$$

where $k^{c}$ and $k_{i}^{l}$ are nonnegative constants.

\section{Full Model}

The assumptions listed above result in the following model of the healthy and the leukemic cell population coupled by the signal $s(t)$ :

$$
\begin{aligned}
\frac{d}{d t} c_{1}(t) & =\left(2 a_{1, \max }^{c} s(t)-1\right) p_{1}^{c} c_{1}(t)-d_{1}^{c} c_{1}(t), \\
\frac{d}{d t} c_{i}(t) & =2\left(1-a_{i-1, \max }^{c} s(t)\right) p_{i-1}^{c} c_{i-1}(t)+\left(2 a_{i, \max }^{c} s(t)-1\right) p_{i}^{c}(t) c_{i}(t)-d_{i}^{c} c_{i}(t), \quad 1<i<n, \\
\frac{d}{d t} c_{n}(t) & =2\left(1-a_{n-1, \max }^{c} s(t)\right) p_{n-1}^{c} c_{n-1}(t)-d_{n}^{c} c_{n}(t), \\
\frac{d}{d t} l_{1}(t) & =\left(2 a_{1, \max }^{l} s(t)-1\right) p_{1}^{l} l_{1}(t)-d_{1}^{l} l_{1}(t), \\
\frac{d}{d t} l_{i}(t) & =2\left(1-a_{i-1, \max }^{l} s(t)\right) p_{i-1}^{l} l_{i-1}(t)+\left(2 a_{i, \max }^{l} s(t)-1\right) p_{i}^{l}(t) l_{i}(t)-d_{i}^{l} l_{i}(t), \quad 1<i<m, \\
\frac{d}{d t} l_{m}(t) & =2\left(1-a_{m-1, \max }^{l} s(t)\right) p_{m-1}^{l} l_{m-1}(t)-d_{m}^{l} l_{m}(t), \\
s(t) & =\frac{1}{1+k^{c} c_{n}(t)+k^{l} l_{m}(t)} .
\end{aligned}
$$

Remark 1. If $l_{1}(0)=\cdots=l_{m}(0)=0$, then $l_{1}(t)=\cdots=l_{m}(t)=0$, for all $t>0$ and the model for healthy hematopoiesis is obtained.

In the following we will analyze which properties of the model of leukemic cells are needed for the leukemic cells present at $t=0$ to destabilize the equilibrium of healthy cells. 


\section{Assumptions}

We make the following assumptions, which are consistent with biological interpretation of respective coefficients:

- $p_{i}^{c}>0$ for $1 \leq i<n, p_{i}^{l}>0$ for $1 \leq i<m$,

- $a_{i}^{c} \in(0,1)$ for $1 \leq i<n, a_{i}^{l} \in(0,1)$ for $1 \leq i<m$,

- $d_{i}^{c} \geq 0$ for $1 \leq i \leq n-1, d_{i}^{l} \geq 0$ for $1 \leq i \leq m-1$,

- $d_{n}^{c}>0, d_{m}^{l}>0$,

- $k^{c}>0, k^{l}>0$,

- $c_{i}(0) \geq 0$ for $1 \leq i \leq n, l_{i}(0) \geq 0$ for $1 \leq i \leq m$.

\section{Analysis}

\subsection{Steady States}

We define healthy hematopoiesis as absence of leukemic cells and existence of a positive steady state of the non-leukemic cell line. Steady state values of $c_{i}$ and $l_{i}$ are denoted as $\bar{c}_{i}$ and $\bar{l}_{i}$, respectively. We consider 3 types of steady states:

Definition 2 (Healthy steady state). Steady state of the form $\bar{c}_{i}>0, i=1, \ldots, n ; \bar{l}_{k}=0, k=$ $1, \ldots, m$ is referred to as the healthy steady state.

Remark 3. The healthy steady state is characterized by the absence of leukemic cells $\left(\bar{l}_{k}=0, k=\right.$ $1, \ldots, m)$. This is an abstract idealization, since in each organism mutations accumulate over lifetime due to replication errors $\left(1\right.$ per $10^{9}$ base pairs per division after repair, or environmental damage: 1 AP site per $10^{5}$ base pairs in steady state of formation and repair), [40]. Mutated cells that may exist in healthy organisms, [35, 61], but that do not lead to formation of relevant neoplastic cell populations are not considered in the above definition.

Definition 4 (Purely leukemic steady state). Steady state of the form $\bar{c}_{i}=0, i=1, \ldots, n$; $\bar{l}_{k}>$ $0, k=1, \ldots, m$ is referred to as the purely leukemic steady state.

Remark 5. The purely leukemic steady state is the abstract reference that cannot be observed in reality since the organism dies in absence of healthy blood cells.

Definition 6 (Composite steady state). Steady state of the form $\bar{c}_{i}>0, i=1, \ldots, n ; \bar{l}_{k}>0, k=$ $1, \ldots, m$, is referred to as the composite steady state. 
We calculate steady states of the system and find parameter conditions for existence of different types of steady states. Our analysis indicates that, the system can have composite steady states or steady states consisting of only healthy or only leukemic cell types. Composite steady states are not unique and they occupy a one-dimensional manifold. This result is summarized in the following Proposition.

Proposition 7 (Steady States).

(i) Assume that there exist $i \in 1, \ldots, m$ and that there exist $j \in 1, \ldots, n$ such that

$$
\frac{\frac{d_{i}^{l}}{p_{i}^{l}}+1}{2 a_{i}^{l}}=\frac{\frac{d_{j}^{c}}{p_{j}^{c}}+1}{2 a_{j}^{c}}:=\bar{s}<1 .
$$

Then, for each $\bar{s}$ with $i, j$ chosen maximal, there exists a one-dimensional manifold of steady states:

$$
\begin{aligned}
& \bar{l}_{m} \in\left[0, \frac{\frac{1}{s}-1}{k^{l}}\right], \bar{c}_{n}=\frac{\frac{1}{\bar{s}}-1-k^{l} \bar{l}_{m}}{k^{c}}, \\
& \bar{l}_{1}=\cdots=\bar{l}_{i-1}=0, \bar{c}_{1}=\cdots=\bar{c}_{j-1}=0 . \\
& \text { For } i \leq k \leq m: \bar{l}_{k}=\bar{l}_{m} \prod_{i=k+1}^{m} \Theta_{i}^{l} \text {, for } j \leq k \leq n: \bar{c}_{k}=\bar{c}_{n} \prod_{i=k+1}^{n} \Theta_{i}^{c}, \text { where } \\
& \Theta_{i}^{l}:=\frac{d_{i}^{l}+p_{i}^{l}-2 a_{i}^{l} p_{i}^{l} \bar{s}}{2\left(1-a_{i-1}^{l} \bar{s}\right) p_{i-1}^{l}}, \Theta_{i}^{c}:=\frac{d_{i}^{c}+p_{i}^{c}-2 a_{i}^{c} p_{i}^{c} \bar{s}}{2\left(1-a_{i-1}^{c} \bar{s} p_{i-1}^{c}\right.}, \Theta_{m}^{l}:=\frac{d_{m}^{l}}{2\left(1-a_{m-1}^{l} \bar{s}\right) p_{m-1}^{l}}, \Theta_{n}^{c}:=\frac{d_{n}^{c}}{2\left(1-a_{n-1}^{c} \bar{s}\right) p_{n-1}^{c}} .
\end{aligned}
$$

There exist also steady states with either $\bar{l}_{i}=0$ for all $i=1, \ldots, m$, or $\bar{c}_{j}=0$ for all $j=1, \ldots, n$. (ii) Assume that for all pairs $i, j,(i=1, \ldots, m ; j=1, \ldots, n)$

$$
\frac{\frac{d_{i}^{l}}{p_{i}^{l}}+1}{2 a_{i}^{l}} \neq \frac{\frac{d_{j}^{c}}{p_{j}^{c}}+1}{2 a_{j}^{c}} .
$$

Then either $\bar{l}_{i}=0$ for all $i=1, \ldots, m$, or $\bar{c}_{j}=0$ for all $j=1, \ldots, n$ in a steady state.

Proof. The result is obtained from calculation of steady states.

We now ask, when the steady states from Proposition 7 are nonnegative.

Proposition 8 (Existence and uniqueness of the healthy steady state). The healthy steady state exists if and only if the following conditions are satisfied:

(i) $\left(2 a_{1}^{c}-1\right) p_{1}^{c}>d_{1}^{c}$

(ii) $\frac{a_{1}^{c} p_{1}^{c}}{d_{1}^{c}+p_{1}^{c}}>\frac{a_{i}^{c} p_{i}^{c}}{d_{i}^{c}+p_{i}^{c}}$

(iii) $d_{n}>0$

If (i)-(iii) are satisfied there exists only one healthy steady state. 
Proof. The proof follows from calculation of steady states imposing $\bar{c}_{i}>0$, for $i=1, \ldots, n$.

Corollary 9. Necessary and sufficient conditions for existence of a purely leukemic steady state are obtained by replacing $p_{i}^{c}, a_{i}^{c}, d_{i}^{c}$ by $p_{i}^{l}, a_{i}^{l}, d_{i}^{l}$.

Proposition 10 (Nonnegative composite steady state).

Assume
(i) $\frac{\frac{d_{1}^{l}}{p_{1}^{l}}+1}{2 a_{1}^{l}}=\frac{\frac{d_{1}^{c}}{p_{1}^{c}}+1}{2 a_{1}^{c}}$
(ii) $\left(2 a_{1}^{c}-1\right) p_{1}^{c}>d_{1}^{c}$
(iii) $\frac{a_{1}^{c} p_{1}^{c}}{d_{1}^{c}+p_{1}^{c}}>\frac{a_{i}^{c} p_{i}^{c}}{d_{i}^{c}+p_{i}^{c}}$
(iv) $d_{n}>0$

Then and only then there exist nonnegative composite steady states.

Proof. The proof follows from calculation of steady states imposing $\bar{c}_{i}>0$, for $i=1, \ldots, n$ and $\bar{l}_{i}>0$, for $i=1, \ldots, m$.

\subsection{Establishment of a Leukemic Stem Cell Population}

In this paragraph we ask, what properties of LSC are necessary for establishment of a leukemic stem cell population. Mathematically speaking, we search for the conditions of instability of $l_{1}(t)$ at the healthy steady state, which we will call instability in the direction of $l_{1}$.

Proposition 11 (Destabilization of the healthy steady state). The healthy steady state is unstable in the direction of $l_{1}$ if and only if

$$
\frac{a_{1}^{l} p_{1}^{l}}{d_{1}^{l}+p_{1}^{l}}>\frac{a_{1}^{c} p_{1}^{c}}{d_{1}^{c}+p_{1}^{c}}
$$

Proof. We linearize the system around the healthy steady state. Instability in the direction of $l_{1}$ depends on the sign of the eigenvalue $\left(2 a_{1}^{l} \bar{s}-1\right) p_{1}^{l}-d_{1}^{l}$ with $\bar{s}:=\frac{d_{1}^{c}+p_{1}^{c}}{2 a_{1}^{c} p_{1}^{c}}$. This eigenvalue is positive if and only if $\frac{a_{1}^{l} p_{1}^{l}}{d_{1}^{l}+p_{1}^{l}}>\frac{a_{1}^{c} p_{1}^{c}}{d_{1}^{c}+p_{1}^{c}}$.

Corollary 12. The healthy steady state is unstable in the direction of $l_{1}$ if and only if the purely leukemic steady state is stable in the direction of $c_{1}$.

Proof. We have $\left(2 a_{1}^{l} \bar{s}^{c}-1\right) p_{1}^{l}-d_{1}^{l}>0$ if and only if $\left(2 a_{1}^{c} \bar{s}^{l}-1\right) p_{1}^{c}-d_{1}^{c}<0$, where $\bar{s}^{c}:=\frac{d_{1}^{c}+p_{1}^{c}}{2 a_{1}^{c} p_{1}^{c}}$ and $\bar{s}^{l}:=\frac{d_{1}^{l}+p_{1}^{l}}{2 a_{1}^{l} p_{1}^{l}}$ denote signal intensity in the healthy steady state and the purely leukemic steady state respectively.

Corollary 13. (i) The following parameter relations are sufficient for instability of the healthy equilibrium in the direction of $l_{1}$ : 
(a) $a_{1}^{l}>a_{1}^{c}, p_{1}^{l}>p_{1}^{c}, d_{1}^{l} \leq d_{1}^{c}$

(b) $a_{1}^{l}=a_{1}^{c}, p_{1}^{l}>p_{1}^{c}, d_{1}^{l}<d_{1}^{c}$

(c) $a_{1}^{l}=a_{1}^{c}, p_{1}^{l}>p_{1}^{c}, d_{1}^{l}=d_{1}^{c}>0$

(d) $a_{1}^{l} \geq a_{1}^{c}, p_{1}^{l} \geq p_{1}^{c}, d_{1}^{l}<d_{1}^{c}$

(e) $a_{1}^{l}>a_{1}^{c}, p_{1}^{l}=p_{1}^{c}, d_{1}^{l}=d_{1}^{c}$

(ii) The parameter relations obtained exchanging superscripts $l$ and c lead to linear stability of the healthy equilibrium in the direction of $l_{1}$.

In the following we give examples of parameter relations leading to growth of a leukemic cell population. The cell properties discussed have been experimentally observed for cells in the leukemic lineages. Nevertheless leukemic stem cells have not yet been observed for all of the provided examples. In the following the most immature cell type of the leukemic cell line is referred to as the leukemic stem cell. This reasoning does not imply that leukemic stem cells have to derive from hematopoietic stem cells. In most of the presented cases the leukemogenic modification occurs on the level of committed precursors.

Example 14. $a_{1}^{l}>a_{1}^{c}, p_{1}^{l}=p_{1}^{c}, d_{1}^{l}=d_{1}^{c}$

- Self-renewal in the leukemic cell line is enhanced in comparison to healthy cells due to mutation or impairment of differentiation. The latter is very common for leukemias and therapeutically exploited in acute promyelocytic leukemia, [1].

Example 15. $a_{1}^{l}=a_{1}^{c}, p_{1}^{l}=p_{1}^{c}, d_{1}^{l}<d_{1}^{c}$

- Scenario 1: Reduced apoptosis in leukemia cells due to mutations (as observed in B-CLL, [53])

- Scenario 2: Induction of apoptosis in healthy cells by malignant cells (as observed in myelodysplastic syndromes, [67])

Example 16. $a_{1}^{l}=a_{1}^{c}, p_{1}^{l}>p_{1}^{c}, d_{1}^{l}=d_{1}^{c} \neq 0$

- Proliferation in malignant cells is enhanced in comparison to healthy cells (as observed in Burkitt-Lymphoma, [12])

Remark 17 (Importance of self-renewal for evolution of leukemia). The case $a_{1}^{l}=a_{1}^{c}, p_{1}^{l}>p_{1}^{c}$, $d_{1}^{l}=d_{1}^{c}=0$ necessarily leads to existence of multiple composite steady states, as discussed in Proposition 7 (i). However, the case $a_{1}^{l}>a_{1}^{c}, p_{1}^{l}=p_{1}^{c}, d_{1}^{l}=d_{1}^{c} \geq 0$ does not. Since composite steady states may cause less severe symptoms, this result demonstrates that changes in differentiation may have more severe consequences than changes in proliferation. 
Remark 18 (Candidate treatment strategies). Corollary 13 (ii) describes qualitative strategies leading to the extinction of small populations of leukemic stem cells. Such strategies are, e.g., reduction of self-renewal of leukemic cells, enhancement of self-renewal of healthy cells, enhanced death of leukemic cells, reduced death of stem cells, enhanced proliferation of stem cells, reduced proliferation of leukemic cells. It depends on the coefficients of the model of leukemic and healthy stem cells if one of these approaches is sufficient or if a combination is required to accomplish that $l_{i} \rightarrow 0$, as $t \rightarrow \infty, i=1, \ldots, m$. Parameter relations that lead to the extinction of leukemic cells are necessary to obtain the complete remission. An important issue in the applications is the rate of leukemic cell elimination, which depends on the values of model parameters. In some cases it might be slow and, therefore, not relevant for the time span corresponding to a realistic lifetime. Therefore, the given scenarios are candidate treatment strategies.

Remark 19 (A more general condition for destabilization). Let us consider a more general system, where $a_{i}^{c}, p_{i}^{c}, d_{i}^{c}$ are arbitrary functions. Denote by $\bar{a}_{1}^{c}, \bar{p}_{1}^{c}, \bar{d}_{1}^{c}$ the values of $a_{i}^{c}, p_{i}^{c}, d_{i}^{c}$ in the healthy steady state. Then it holds $\left(2 \bar{a}_{1}^{c}-1\right) \bar{p}_{1}^{c}-\bar{d}_{1}^{c}=0$. Let $\bar{a}_{i}^{l}, \bar{p}_{i}^{l}, \bar{d}_{i}^{l}$, denote the values of $a_{i}^{l}, p_{i}^{l}, d_{i}^{l}$ in the healthy steady state. Then it is necessary and sufficient for destabilization of the healthy steady state that $\left(2 \bar{a}_{1}^{l}-1\right) \bar{p}_{1}^{l}-\bar{d}_{1}^{l}>0$, i.e. $\left(2 \bar{a}_{1}^{l}-1\right) \bar{p}_{1}^{l}-\bar{d}_{1}^{l}>\left(2 \bar{a}_{1}^{c}-1\right) \bar{p}_{1}^{c}-\bar{d}_{1}^{c}$, which is equivalent to $\left(2 \bar{a}_{1}^{l}-1\right) \bar{p}_{1}^{l}-\left(2 \bar{a}_{1}^{c}-1\right) \bar{p}_{1}^{c}>\bar{d}^{l}-\bar{d}^{c}$. The examples listed above also fulfill this more general condition.

\subsection{System with 4 equations}

The results about destabilization of the healthy steady state holds for arbitrary compartment numbers. To obtain insight into dynamics of the considered system, we perform linear stability analysis. Due to technical complexity of large systems, we focus on the case $n=2, m=2$ in the remainder of this paper. This is the minimal set of equations describing the healthy and leukemic hematopoiesis. Numerical simulations for wide parameter ranges suggest that a reduced model consisting only of two differentiation steps for each cell lineage shows dynamics qualitatively similar to the models consisting of more equations.

Proposition 20 (Linear stability of purely leukemic steady state).

Assume

(i) $\frac{d_{1}^{c}+p_{1}^{c}}{a_{1}^{c} p_{1}^{c}}>\frac{d_{1}^{l}+p_{1}^{l}}{a_{1}^{l} p_{1}^{l}}$

(ii) $\left(2 a_{1}^{c}-1\right) p_{1}^{c}>d_{1}^{c}$

(iii) $d_{2}^{c}>0, d_{2}^{l}>0$.

Then, there exists a unique healthy and a unique purely leukemic steady state. The purely leukemic steady state is locally stable and the healthy steady state is unstable in the direction of $l_{1}$.

Remark 21. Assumption (i) is necessary and sufficient for establishment of a leukemic cell line, see Proposition 11. Assumptions (ii) and (iii) are necessary and sufficient for existence of a healthy steady state, see Proposition 8. 
Proof. The existence of a unique healthy steady state follows from Proposition 8. Assumption (ii) is equivalent to $\frac{2 a_{1}^{c} p_{1}^{c}}{d_{1}^{c}+p_{1}^{c}}>1$. Assumption (i) implies $\frac{2 a_{1}^{l} p_{1}^{l}}{d_{1}^{l}+p_{1}^{l}}>1$ and therefore $\left(2 a_{1}^{l}-1\right) p_{1}^{l}>d_{1}^{l}$. Due to the symmetry of the considered ODE system with respect to interchange of $c_{i}$ and $l_{i}$ this and application of Proposition 8 lead to existence and uniqueness of the purely leukemic steady state.

Since $\bar{c}_{1}=\bar{c}_{2}=0$ and, due to the steady state condition, $\left(2 a_{1}^{l} \bar{s}-1\right) p_{1}^{l}-d_{1}^{l}=0$, with $\bar{s}:=\frac{d_{1}^{l}+p_{1}^{l}}{2 a_{1}^{l} p_{1}^{l}}$, the characteristic polynomial of the linearization around the purely leukemic steady state calculates as:

$$
\begin{aligned}
\chi(\lambda)= & \left|\begin{array}{cc}
\lambda & 2 a_{1}^{l} p_{1}^{l} k^{l} \bar{s}^{2} \bar{l}_{1} \\
-2\left(1-a_{1}^{l} \bar{s}\right) p_{1}^{l} & \lambda-\left(2 a_{1}^{l} p_{1}^{l} k^{l} \bar{s}^{2} \bar{l}_{1}-d_{2}^{l}\right)
\end{array}\right|\left(\lambda+d_{2}^{c}\right)\left(\lambda-\left[\left(2 a_{1}^{c} \bar{s}-1\right) p_{1}^{c}-d_{1}^{c}\right]\right) \\
= & {\left[\begin{array}{c}
\left.\lambda^{2}-\left(\frac{d_{2}^{l}}{p_{1}^{l}-d_{1}^{l}} \frac{d_{1}^{l}+p_{1}^{l}}{\left(2 a_{1}^{l} p_{1}^{l}\right)}\left(\left(2 a_{1}^{l}-1\right) p_{1}^{l}-d_{1}^{l}\right)-d_{2}^{l}\right) \lambda+4 a_{1}^{l}\left(p_{1}^{l}\right)^{2} k^{l} \bar{s}^{2}\left(1-a_{1}^{l} \bar{s}\right) \bar{l}_{1}\right] \\
\cdot\left(\lambda+d_{2}^{c}\right)\left(\lambda-\left[\left(2 a_{1}^{c} \bar{s}-1\right) p_{1}^{c}-d_{1}^{c}\right]\right)
\end{array}\right.}
\end{aligned}
$$

with $\bar{s}:=\frac{d_{1}^{l}+p_{1}^{l}}{2 a_{1}^{l} p_{1}^{l}}<1$, due to conditions (i) and (ii) and $\bar{l}_{1}=\frac{d_{2}^{l}(1 / \bar{s}-1)}{2\left(1-a_{1}^{l} \bar{s}\right) p_{1}^{l} k^{l}}$. Therefore $4 a_{1}^{l}\left(p_{1}^{l}\right)^{2} k^{l} \bar{s}^{2}(1-$ $\left.a_{1}^{l} \bar{s}\right) \bar{l}_{1}>0$. It holds that $2 a_{1}^{l} p_{1}^{l}-d_{1}^{l}-p_{1}^{l}<2 p_{1}^{l}-d_{1}^{l}-p_{1}^{l}=p_{1}^{l}-d_{1}^{l}$, since $a_{1}^{l}<1$. This implies

$$
\begin{aligned}
\frac{d_{2}^{l}}{p_{1}^{l}-d_{1}^{l}} \frac{d_{1}^{l}+p_{1}^{l}}{\left(2 a_{1}^{l} p_{1}^{l}\right)}\left(\left(2 a_{1}^{l}-1\right) p_{1}^{l}-d_{1}^{l}\right)-d_{2}^{l} & <\frac{d_{2}^{l}}{p_{1}^{l}-d_{1}^{l}} \frac{d_{1}^{l}+p_{1}^{l}}{\left(2 a_{1}^{l} p_{1}^{l}\right)}\left(p_{1}^{l}-d_{1}^{l}\right)-d_{2}^{l} \\
& =d_{2}^{l}(\bar{s}-1) \\
& <0 .
\end{aligned}
$$

Therefore

$-\left(\frac{d_{2}^{l}}{p_{1}^{l}-d_{1}^{l}} \frac{d_{1}^{l}+p_{1}^{l}}{\left(2 a_{1}^{l} p_{1}^{l}\right)}\left(\left(2 a_{1}^{l}-1\right) p_{1}-d_{1}^{l}\right)-d_{2}^{l}\right)>0$. Vieta's theorem implies that all eigenvalues of the quadratic polynomial have negative real part. Since $\left(2 a_{1}^{c} \bar{s}-1\right) p_{1}^{c}-d_{1}^{c}<0$, due to (i), all eigenvalues of the linearization have negative real part.

Corollary 22 (Linear stability of healthy steady state). Renaming variables $\left(p_{i}^{c} \leftrightarrow p_{i}^{l}, a_{i}^{c} \leftrightarrow a_{i}^{l}\right.$, $c_{i} \leftrightarrow l_{i}^{l}$ ) in Proposition 20 leads to the existence of a unique healthy and a unique purely leukemic steady state and linear stability of the healthy steady state.

\subsection{Linearized stability analysis of composite steady states}

In this Section we study stability of composite steady states. For simplicity of calculations we set $k^{l}=k^{c}=: k$ in the following Section. Let the assumptions of Propositions 7 (i) and 10 hold. We linearize around the composite steady state discussed in Proposition 7 (i). The linearization $M$ calculates

$$
M:=\left|\begin{array}{cccc}
0 & -\alpha^{c} & 0 & -\alpha^{c} \\
\nu^{c} & \rho^{c} & 0 & \alpha^{c} \\
0 & -\alpha^{l} & 0 & -\alpha^{l} \\
0 & \alpha^{l} & \nu^{l} & \rho^{l}
\end{array}\right|,
$$


where

$$
\begin{aligned}
\alpha^{c} & :=2 a_{1}^{c} k p_{1}^{c} \bar{c}_{1} \bar{s}^{2}>0, \\
\nu^{c} & :=2\left(1-a_{1}^{c} \bar{s}\right) p_{1}^{c}>0, \\
\rho^{c} & :=\alpha^{c}-d_{2}^{c}, \\
\alpha^{l} & :=2 a_{1}^{l} k p_{1}^{l} \bar{l}_{1} \bar{s}^{2}>0, \\
\nu^{l} & :=2\left(1-a_{1}^{l} \bar{s}\right) p_{1}^{l}>0, \\
\rho^{l} & :=\alpha^{l}-d_{2}^{l} .
\end{aligned}
$$

Then the characteristic polynomial of the linearization around the composite steady state is of the form:

$$
\chi_{M}(\lambda):=\left(\lambda^{3}+\left(-\rho^{l}-\rho^{c}\right) \lambda^{2}+\left(-\alpha^{l} \alpha^{c}+\nu^{l} \alpha^{l}+\rho^{l} \rho^{c}+\nu^{c} \alpha^{c}\right) \lambda+\nu^{c} \alpha^{c}\left(\alpha^{l}-\rho^{l}\right)+\nu^{l} \alpha^{l}\left(\alpha^{c}-\rho^{c}\right)\right) \lambda .
$$

Remark 23. We observe that $\chi_{M}$ has one zero eigenvalue.

Proposition 24 (Center Manifold). If there exists only one eigenvalue with zero real part, then the manifold of the steady states is a center manifold.

The proof is presented in the Appendix.

We continue to analyze the eigenvalues of

$$
\tilde{\chi}_{M}(\lambda):=\lambda^{3}+\left(-\rho^{l}-\rho^{c}\right) \lambda^{2}+\left(-\alpha^{l} \alpha^{c}+\nu^{l} \alpha^{l}+\rho^{l} \rho^{c}+\nu^{c} \alpha^{c}\right) \lambda+\nu^{c} \alpha^{c}\left(\alpha^{l}-\rho^{l}\right)+\nu^{l} \alpha^{l}\left(\alpha^{c}-\rho^{c}\right) .
$$

We intend to use the Routh-Hurwitz-Criterion, [29], and therefore calculate Hurwitz determinants of $\tilde{\chi}_{M}(\lambda)$.

Lemma 25. Let $\tilde{\chi}_{M}(\lambda):=\lambda^{3}+\left(-\rho^{l}-\rho^{c}\right) \lambda^{2}+\left(-\alpha^{l} \alpha^{c}+\nu^{l} \alpha^{l}+\rho^{l} \rho^{c}+\nu^{c} \alpha^{c}\right) \lambda+\nu^{c} \alpha^{c}\left(\alpha^{l}-\right.$ $\left.\rho^{l}\right)+\nu^{l} \alpha^{l}\left(\alpha^{c}-\rho^{c}\right):=\lambda^{3}+a_{2} \lambda^{2}+a_{1} \lambda+a_{0}$. It holds:

(i) The absolute term $a_{0}$ of $\tilde{\chi}_{M}(\lambda)$ is positive.

(ii) The quadratic term $a_{2}$ of $\tilde{\chi}_{M}(\lambda)$ is positive.

(iii) Assume $k:=k^{l}=k^{c}$ and $d_{1}^{c}=d_{1}^{l}=0$, then the second determinant of Hurwitz $H_{2}:=$ $a_{2} a_{1}-a_{3} a_{0}$ can be written as

$$
H_{2}(X)=\tilde{\alpha} X^{2}+\tilde{\beta} X+\tilde{\gamma},
$$

where

$$
\begin{aligned}
& X:=k \bar{l}_{2}, \\
& \tilde{\alpha}:=\hat{\alpha} \bar{s}^{2}, \text { with } \hat{\alpha}:=\left(p_{1}^{l} d_{2}^{l}-p_{1}^{c} d_{2}^{c}\right)\left(d_{2}^{c}-d_{2}^{l}\right),
\end{aligned}
$$




$$
\begin{aligned}
& \tilde{\beta}:=-\bar{s}(1-\bar{s}) \hat{\alpha}+\bar{s}^{2}\left[p_{1}^{l}\left(d_{2}^{l}\right)^{2}-p_{1}^{c}\left(d_{2}^{c}\right)^{2}+d_{2}^{c} d_{2}^{l}\left(d_{2}^{c}-d_{2}^{l}\right)\right], \\
& \tilde{\gamma}:=d_{2}^{c}\left(d_{2}^{l}\right)^{2} \bar{s}+\left(d_{2}^{c}\right)^{2} d_{2}^{l} \bar{s}^{2}+\left(d_{2}^{c}\right)^{2} p_{1}^{c}\left(2 a_{1}^{c}-1\right) \bar{s}^{2}>0 .
\end{aligned}
$$

The proof is presented in the Appendix. For the remainder of this Section we assume $d_{1}^{l}=d_{1}^{c}=0$, for simplicity.

We now ask which range of parameters is biologically relevant. Since $\bar{s}=\frac{1}{2 a_{1}^{c}}=\frac{1}{1+k \bar{l}_{2}+k \bar{c}_{2}}$ with $\bar{c}_{2} \geq 0, \bar{l}_{2} \geq 0$, it is necessary that $k \bar{l}_{2}$ is $0 \leq k \bar{l}_{2} \leq\left(2 a_{1}^{c}-1\right)$. Since we assume $a_{1}^{c}<1$ it follows $\frac{1}{2}<\bar{s}=\frac{1}{2 a_{1}^{c}}=\frac{1}{1+k \bar{l}_{2}+k \bar{c}_{2}}<1$. This motivates the following Definition.

Definition 26 (Biologically relevant range of parameters). Let $a_{i}^{c}, p_{i}^{c}, d_{i}^{c}, k^{l}, k^{c}, a_{i}^{l}, p_{i}^{l}, d_{i}^{l}$ fulfill the assumptions specified in Section 1, then the following range of parameters is referred to as biologically relevant range of parameters:

- $\frac{1}{2}<\bar{s}<1$,

- $0 \leq k \bar{l}_{2} \leq\left(2 a_{1}^{c}-1\right), 0 \leq k \bar{c}_{2} \leq\left(2 a_{1}^{c}-1\right)$.

In the following we investigate the signum of $H_{2}(X)$ within the ranges of this Definition.

Proposition 27. If $\hat{\alpha}<0$ it holds $H_{2}(X)>0$ in the biologically relevant range of parameters.

The proof is presented in the Appendix.

Remark 28 (Biological interpretation). It holds $\hat{\alpha}<0$ if and only if either $\left(p_{1}^{l} d_{2}^{l}>p_{1}^{c} d_{2}^{c}\right.$ and $\left.d_{2}^{c}<d_{2}^{l}\right)$ or $\left(p_{1}^{l} d_{2}^{l}<p_{1}^{c} d_{2}^{c}\right.$ and $\left.d_{2}^{c}>d_{2}^{l}\right)$. If $d_{2}^{c}<d_{2}^{l}$ and $p_{1}^{c}$ is small enough or $p_{1}^{l}$ is large enough, the system is stable in the sense that it asymptotically approaches the center manifold if it starts in the neighborhood of it, the same if $d_{2}^{c}>d_{2}^{l}$ and $p_{1}^{l}$ is small enough or $p_{1}^{c}$ is large enough.

Proposition 29. If $\hat{\alpha}=0$ it holds $H_{2}(X)>0$ in the biologically relevant range of parameters.

The proof is presented in the Appendix.

Remark 30 (Biological interpretation). It holds $\hat{\alpha}=0$ if and only if either $p_{1}^{l} d_{2}^{l}=p_{1}^{c} d_{2}^{c}$ or $d_{2}^{c}=d_{2}^{l}$. In these cases the system is stable in the sense that it asymptotically approaches the center manifold if it starts in the neighborhood of it.

Proposition 31. (i) If $\hat{\alpha}>0$ and $k \bar{l}_{2}$ small enough, then the system is stable in the sense that it asymptotically approaches the center manifold if it starts in the neighborhood of it. If $\hat{\alpha} \leq 0$ it is stable in that sense.

(ii) For $\hat{\alpha}>0$ there exists a parameter range, where the system becomes unstable.

The proof and a characterization of the corresponding parameter ranges are presented in the Appendix. 
Remark 32. For a long time there exists the hypothesis that instability and chaos may play a role in cancer formation [7, 21, 9]. Mathematical studies and experimental evidence suggest that the hematopoietic system may exhibit chaos and instability [3, 45, 60]. The above finding of instability is therefore in line with results from literature.

Example 33. Numerical solutions with parameters from the instable range are shown in Figure 6 for different time scales. For comparison, a numerical solution with parameters of linear stable coexistence and perturbations of the same relative order of magnitude is shown in Figure 5.

Remark 34 (Biological Interpretation). (i) The fact that there exists no instability for $k \bar{l}_{2}$ small enough might explain, while some syndromes with small amounts of malignant cells, such as myelodysplasic syndromes or MGUS, are stable over years.

(ii) The necessary condition $\hat{\alpha}>0$ is equivalent to $d_{2}^{c}>d_{2}^{l}$ and $p_{1}^{l} d_{2}^{l}>p_{1}^{c} d_{2}^{c}$. This implies $p_{1}^{l}>p_{1}^{c}$. These conditions are synergistic in the sense that the cell line that dies slower shows stronger proliferation of stem cells. Such synergism is necessary for the emergence of instability. The necessary condition obtained after renaming of variables lead to the same result.

(iii) The proposition shows that instability of coexistence emerges only for some parameter ranges. If the proliferation rate of stem cells, $p_{1}^{c}$, is large enough, coexistence is linearly stable. That might lead to the conclusion that stimulation of healthy stem cells stabilizes preleukemic states.

\section{Numerical Solutions}

This Section is devoted to presentation of the numerical solutions of important cases of the proposed model. Numerics are based on the explicit Runge-Kutta Method. To obtain insight into the dynamics, $p_{1}^{c}$ has been rescaled to 1 and the number of healthy mature cells to values between $10^{9}$ and $10^{10}$. Numerical simulations demonstrate that for wide parameter ranges dynamics of systems with more compartments are qualitatively similar to the presented case. In the $2 \times 2$ case, the first compartment corresponds to a mixture of stem- and progenitor cells.

\subsection{Purely leukemic steady state}

Figure 1 gives an example of the case where leukemic stem cells have enhanced self-renewal potential and decreased proliferative activity compared to healthy stem cells. Numerical solutions show a clinically insignificant phase with normal healthy and low leukemic cell counts followed by a sudden rise of leukemic and a sudden reduction of healthy cell counts. This behavior is comparable to this seen in acute leukemias, such as AML. 

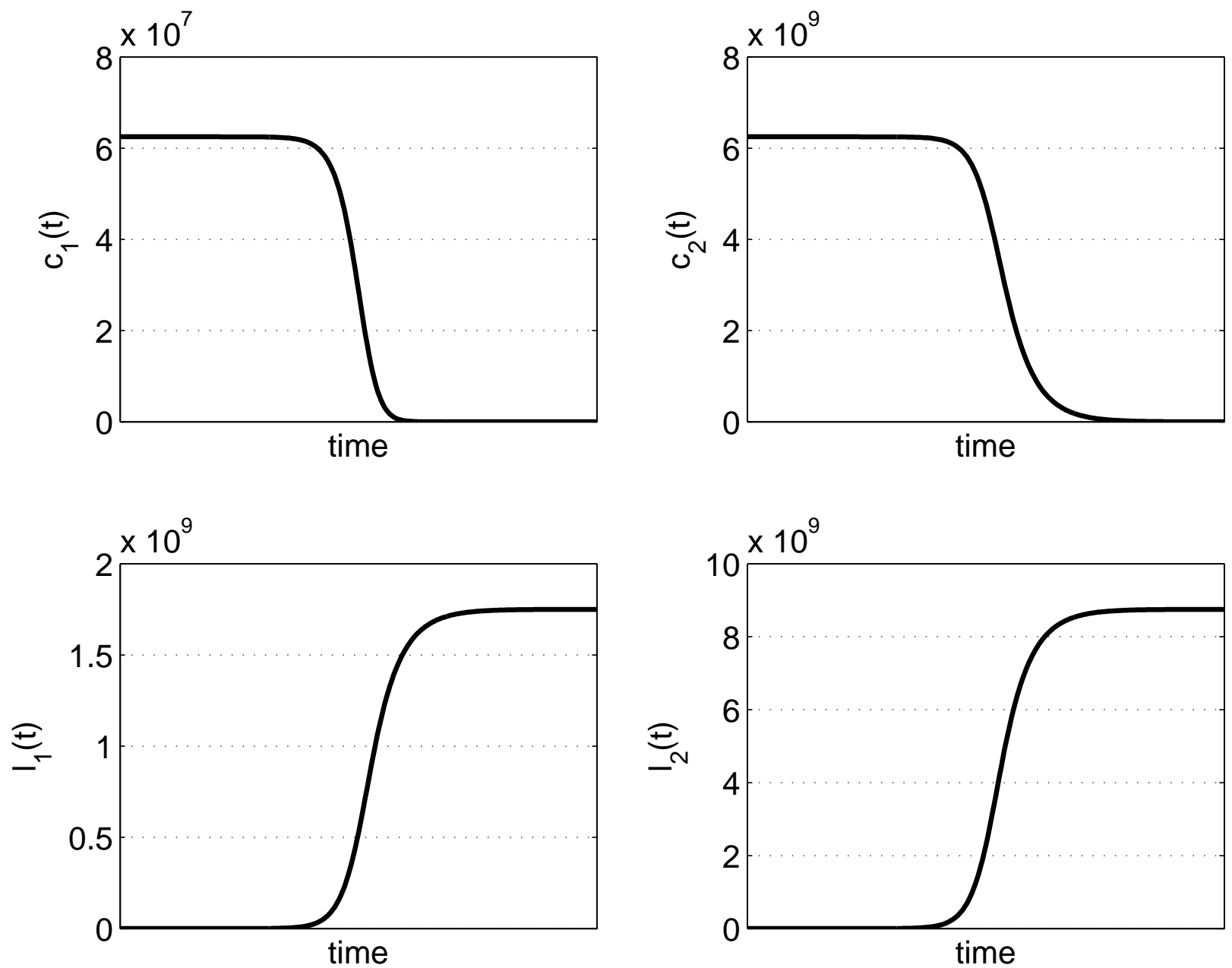

Figure 1: Establishment of a leukemic steady state and extinction of healthy cells: Leukemia establishes although proliferation of leukemic cells is smaller than proliferation of healthy cells. Parameters: $a_{1}^{c}=0.55, p_{1}^{c}=1, d_{1}^{c}=0, d_{2}^{c}=0.01 ; a_{1}^{l}=0.57, p_{1}^{l}=0.5, d_{1}^{l}=0, d_{2}^{l}=0.1, k=$ $1.6 \cdot 10^{-11}$. Initial conditions: Healthy cell levels are values of healthy equilibrium, $l_{1}(0)=10$, $l_{2}(0)=0$. 


\subsection{Re-establishment of the healthy steady state}

Figure 2 gives an example of the extinction of a mutated cell clone. In comparison to the healthy stem cells, the mutated cells show reduced self-renewal and enhanced proliferation. In this example, enhanced proliferation is not sufficient to compensate for reduced self-renewal potential. This example demonstrates the complex interplay of different cell properties that is necessary for persistence of the mutant clone. The numerical solution shows decrease of the mutant cell clone in a relatively short time scale. This scenario describes a benign mutation. The changes of healthy cell counts are negligible on the clinically relevant scale.
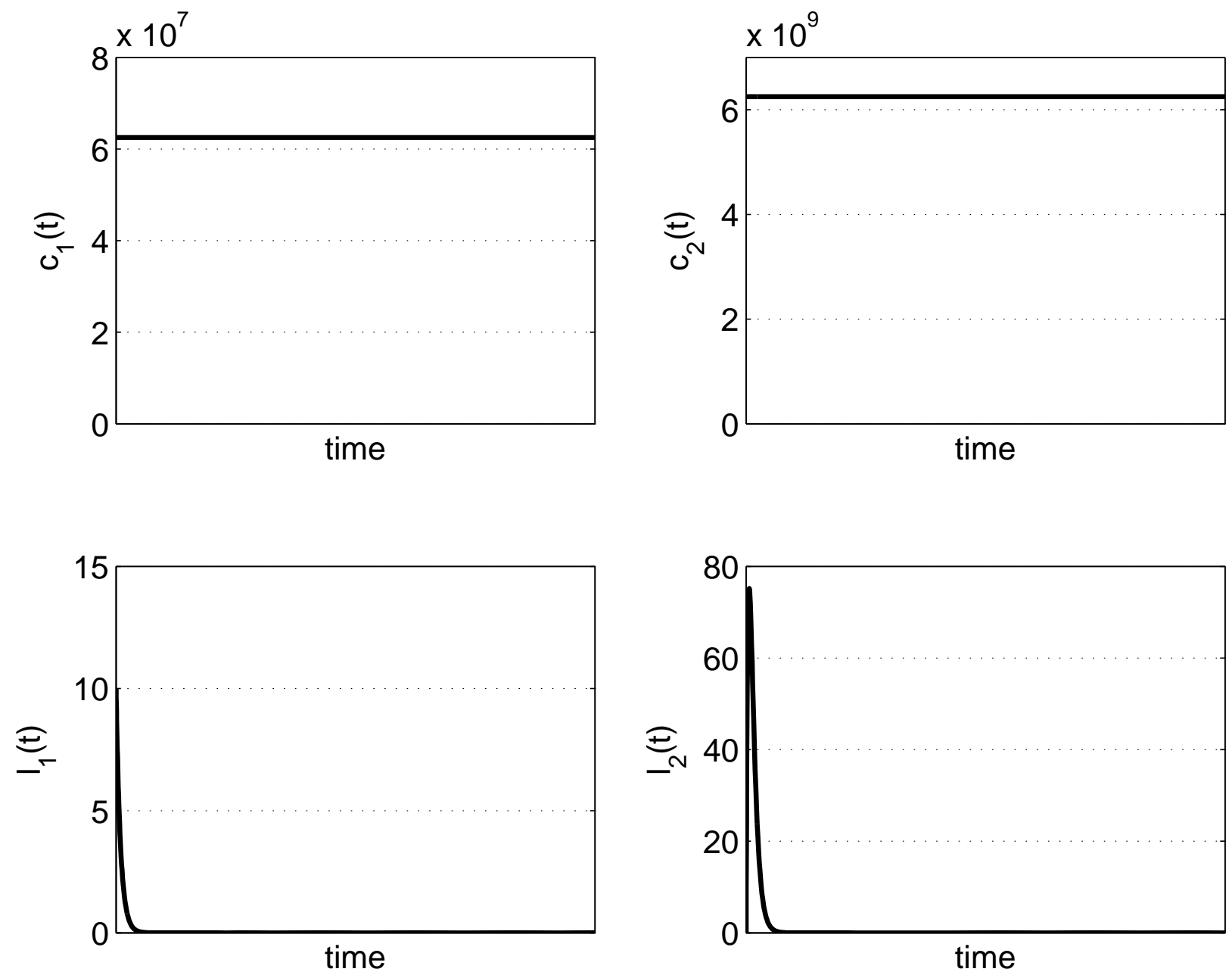

Figure 2: Extinction of the leukemic cell population and reestablishment of healthy equilibrium: Stability of healthy steady state. Parameters: $a_{1}^{c}=0.55, p_{1}^{c}=1, d_{1}^{c}=0, d_{2}^{c}=0.01 ; a_{1}^{l}=$ $0.53, p_{1}^{l}=1.5, d_{1}^{l}=0, d_{2}^{l}=0.1, k=1.6 \cdot 10^{-11}$. Initial conditions: Healthy cell levels are values of healthy equilibrium, $l_{1}(0)=10, l_{2}(0)=0$. 


\subsection{Coexistence}

Figure 3 depicts the coexistence of healthy and leukemic cell populations in equilibrium. Numerical solutions show a moderate and slow change of healthy cell counts to a new equilibrium. This scenario is comparable to preleukemic states, myelodysplasias, monoclonal gammopathy of unknown significance or the so-called smoldering myelomas $[38,10]$ that can persist for years without major impairment of blood function. Transformation to leukemia is possible if parameters change in favor of the leukemic population, e.g., by mutation and selection. Re-establishment of the healthy equilibrium is possible if parameters change in favor of the healthy population. Since this scenario requires a sharp condition on the parameters, it can be considered a rare case.
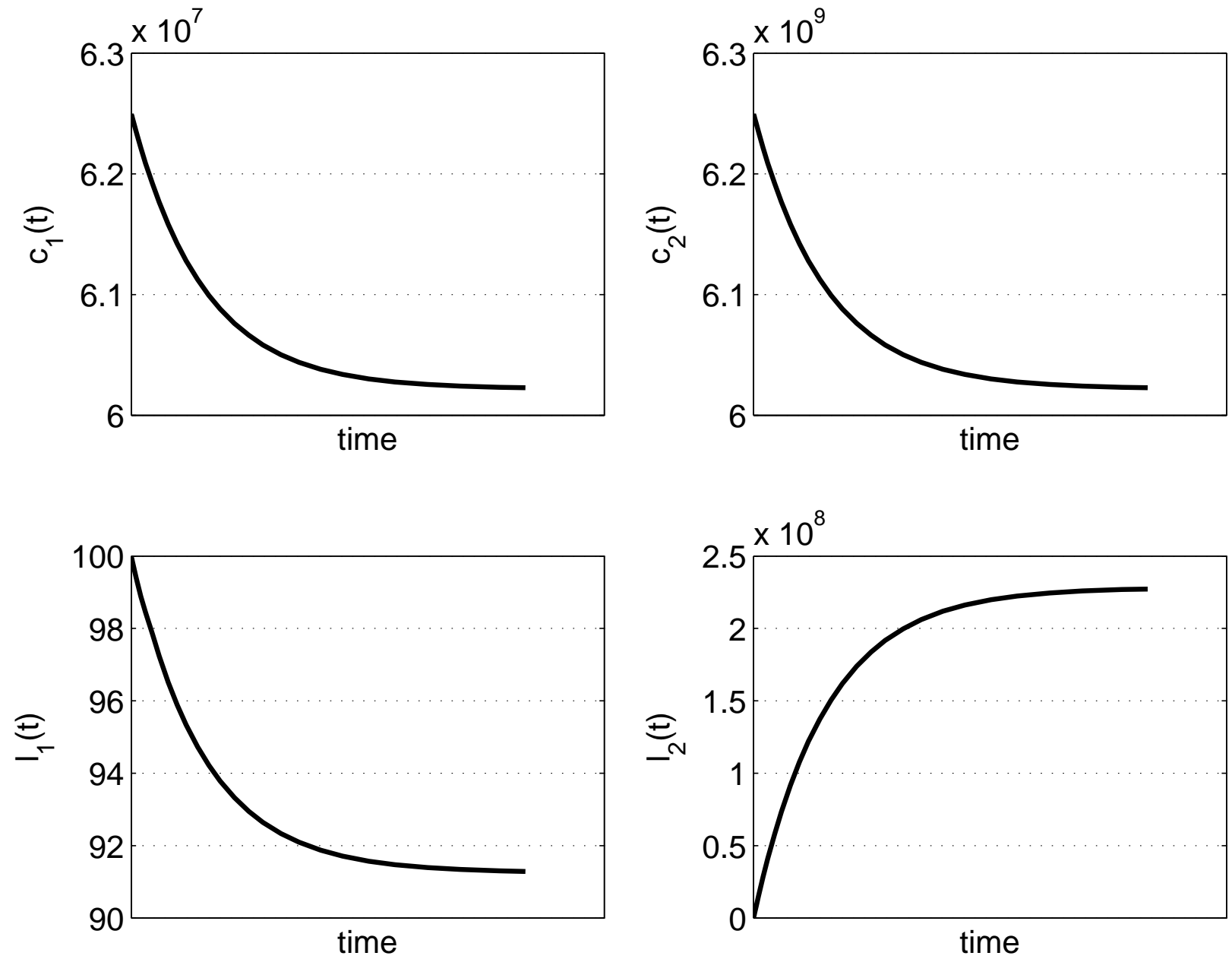

Figure 3: Equilibrium with coexistence of healthy and leukemic cells: The leukemic cell count is in the order of magnitude of the count of healthy mature cells. Parameters: $a_{1}^{c}=0.55, p_{1}^{c}=$ $1, d_{1}^{c}=0, d_{2}^{c}=0.01 ; a_{1}^{l}=a_{1}^{c}, p_{1}^{l}=2.5, d_{1}^{l}=0, d_{2}^{l}=10 \cdot 10^{-6}, k=1.6 \cdot 10^{-11}$. Initial conditions: Healthy cell levels are values of healthy equilibrium, $l_{1}(0)=100, l_{2}(0)=0$. 
Figure 4 shows coexistence of leukemic and healthy cells in equilibrium without a measurable change of healthy cell counts. This scenario may describe the existence of sub-clinical mutated cell-lines that can be found in the marrow of healthy individuals $[35,61]$.
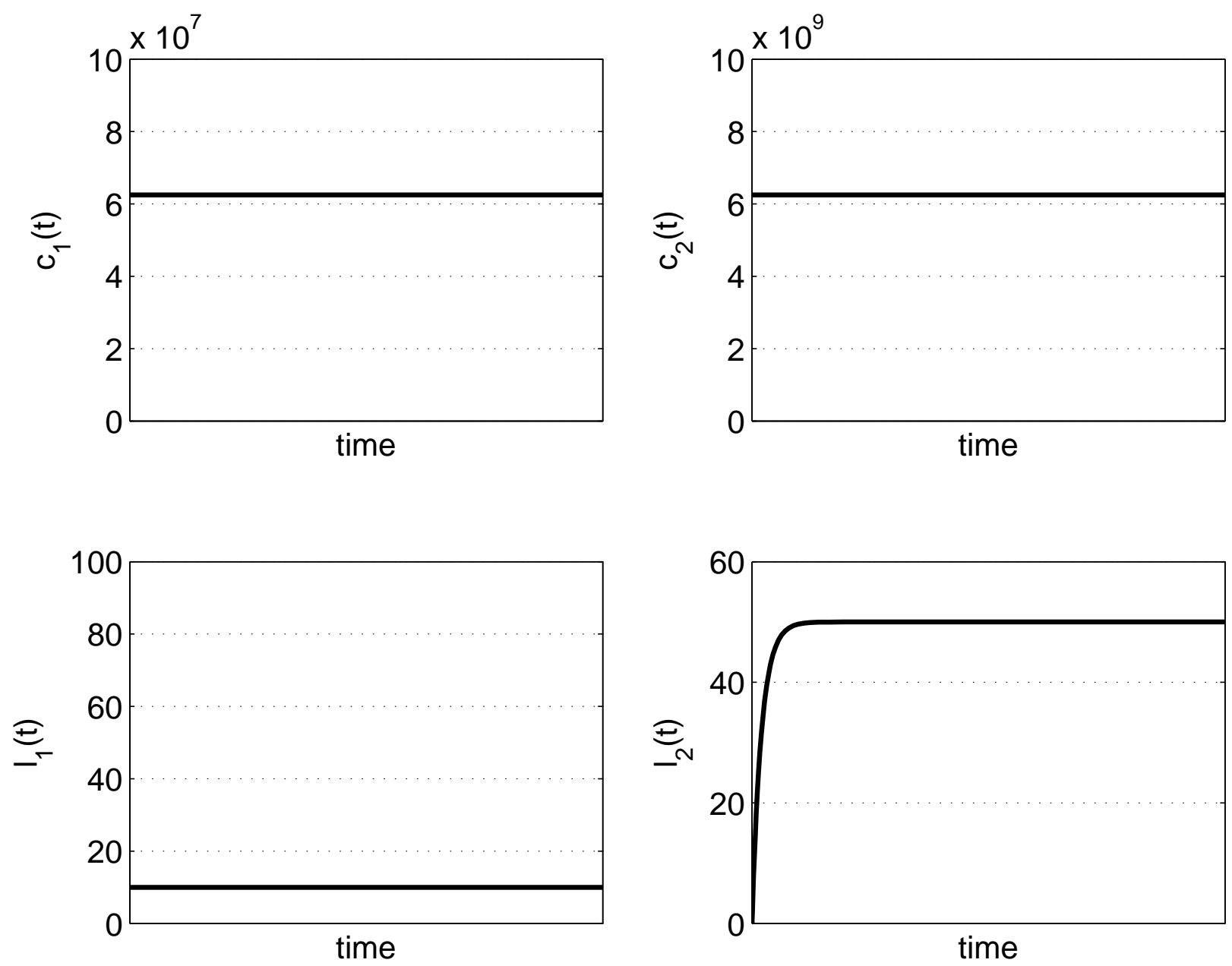

Figure 4: Equilibrium with coexistence of healthy and leukemic cells: The leukemic cell count is small and the change of healthy cell counts is invisible on a clinically relevant scale. Parameters: $a_{1}^{c}=0.55, p_{1}^{c}=1, d_{1}^{c}=0, d_{2}^{c}=0.01 ; a_{1}^{l}=a_{1}^{c}, p_{1}^{l}=0.5, d_{1}^{l}=0, d_{2}^{l}=0.1, k=1.6 \cdot 10^{-11}$. Initial conditions: Healthy cell levels are values of healthy equilibrium, $l_{1}(0)=10, l_{2}(0)=0$.

Figures 5 and 6 compare reaction of the system to small perturbations of the composite steady state. Depending on parameters and initial conditions small perturbations of initial conditions lead to convergence to nearby steady-states (see Figure 5), or to convergence to relatively distant steady states (see Figure 6). The latter case occurs for specific parameter conditions only and therefore might not be observed in healthy organisms. 

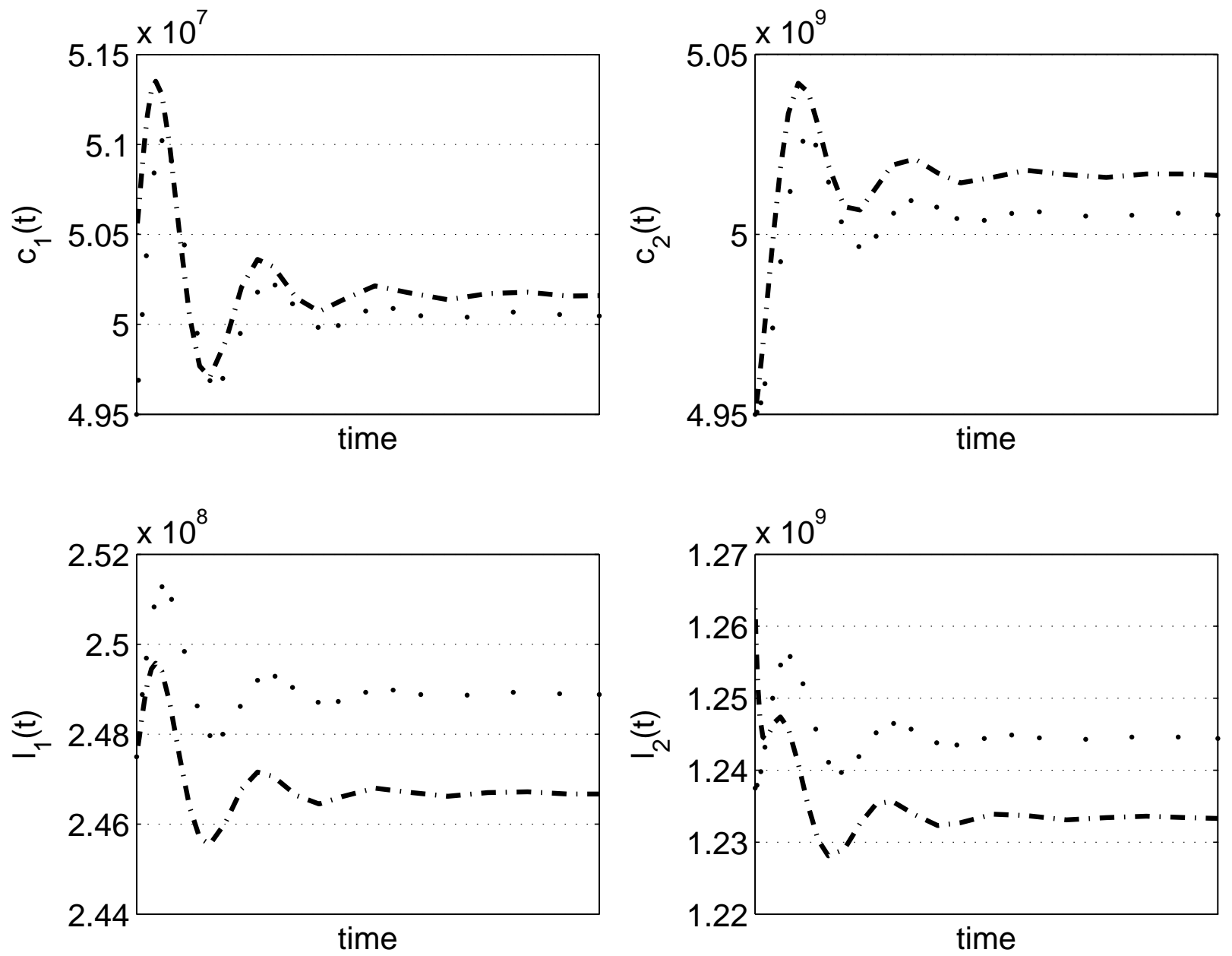

Figure 5: Perturbation of equilibrium with coexistence of healthy and leukemic cells: Small perturbations of points on the manifold of steady states lead to similar time dynamics and steady states. The perturbation is about $1 \%$ of the point $\left(c_{1}^{m}, c_{2}^{m}, l_{1}^{m}, l_{2}^{m}\right)$ on the manifold of steady states characterized by $c_{2}^{m}=0.8 \bar{c}_{2}^{h}$, where $\bar{c}_{2}^{h}$ is the count of mature cells in the healthy equilibrium. Parameters: $a_{1}^{c}=0.55, p_{1}^{c}=1, d_{1}^{c}=0, d_{2}^{c}=0.01 ; a_{1}^{l}=a_{1}^{c}, p_{1}^{l}=0.5, d_{1}^{l}=0, d_{2}^{l}=0.1, k=$ $1.6 \cdot 10^{-11}$. Initial conditions: $c_{1}(0)=1.01 \cdot c_{1}^{m}, c_{2}(0)=0.99 \cdot c_{2}^{m}, l_{1}(0)=0.99 \cdot l_{1}^{m}, l_{2}(0)=1.01 \cdot l_{2}^{m}$ or $c_{1}(0)=0.99 \cdot c_{1}^{m}, c_{2}(0)=0.99 \cdot c_{2}^{m}, l_{1}(0)=0.99 \cdot l_{1}^{m}, l_{2}(0)=0.99 \cdot l_{2}^{m}$ resp. 

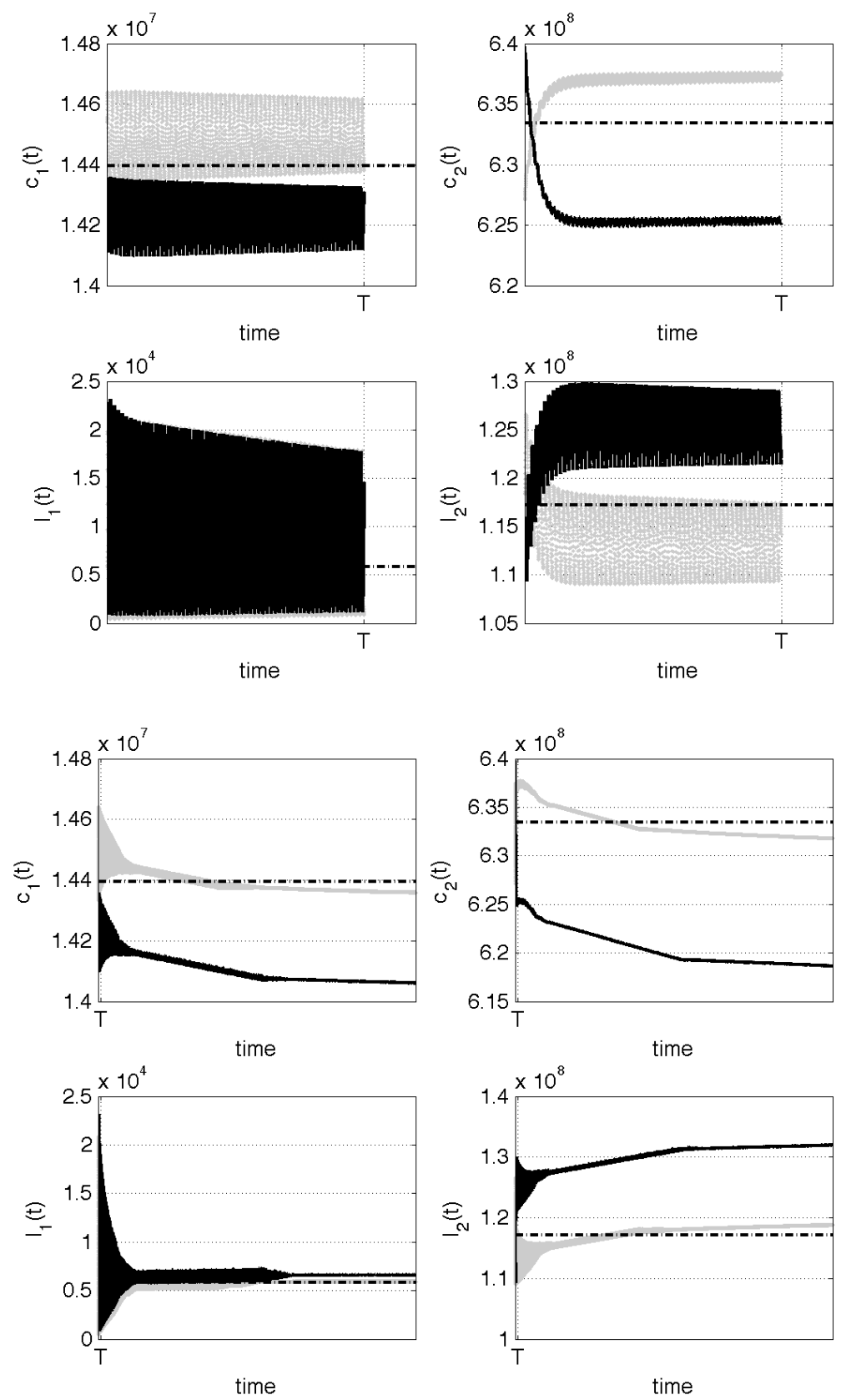

Figure 6: Perturbation of equilibrium with coexistence of healthy and leukemic cells: Small perturbations lead to oscillations and steady states relatively far away from each other. The perturbation is about $1 \%$ of the point $\left(c_{1}^{m}, c_{2}^{m}, l_{1}^{m}, l_{2}^{m}\right)$ on the manifold of steady states characterized by $l_{2}^{m}=0.15 / k$. Parameters: $a_{1}^{c}=0.98039, p_{1}^{c}=22, d_{1}^{c}=0, d_{2}^{c}=0.5, a_{1}^{l}=a_{1}^{c}, p_{1}^{l}=4000, d_{1}^{l}=0$, $d_{2}^{l}=0.2, k=12.8 \cdot 10^{-10}$. Initial conditions: $c_{1}(0)=0.99 \cdot c_{1}^{m}, c_{2}(0)=1.01 \cdot c_{2}^{m}, l_{1}(0)=1.01 \cdot l_{1}^{m}$, $l_{2}(0)=0.99 \cdot l_{2}^{m}$ or $c_{1}(0)=1.01 \cdot c_{1}^{m}, c_{2}(0)=0.99 \cdot c_{2}^{m}, l_{1}(0)=0.99 \cdot l_{1}^{m}, l_{2}(0)=1.01 \cdot l_{2}^{m}$ resp. The above 4 pictures describe behavior of the solution for short time scale, the 4 pictures below for long time scale. The ending point $T$ of time axis in the first pictures is also marked in the pictures showing long time scale behavior. 


\section{Conclusions}

We proposed a mathematical model describing time evolution of a leukemic cell line competing with healthy hematopoiesis. The model is based on qualitative measurable cell properties such as fractions of self-renewal, proliferation rates and death rates of the cell types considered. Interaction of the competing cell lines is modeled using nonlinear negative feedback signaling motivated by cytokine dynamics in hematopoiesis. Tools of linear stability analysis provide sufficient and necessary conditions for destabilization of the healthy equilibrium and expansion of a mutated cell clone. These conditions allow comparing the properties of leukemia initiating cells to those of the physiological adult stem cells.

Different kinds of steady states can appear in the model, depending on the model parameters. In most cases there exists a unique healthy and a unique purely leukemic steady state, but no composite steady states. We performed linear stability analysis for a version of the model including hematopoietic stem cells, mature blood cells, leukemic stem cells and non-stem leukemic cells. In absence of composite steady states, the leukemic steady state is locally stable if the healthy steady state is destabilized in the direction of the leukemic stem cell population.

The appearance of composite steady states is characterized by existence of one zero eigenvalue and a one dimensional manifold of steady states, which is locally equivalent to a center manifold. The composite steady state can be interpreted as a preleukemic state or a chronic disease such as myelodysplastic syndrome or MGUS. There exist composite steady states with positive real parts of some eigenvalues. If the proliferation rate of stem cells is large enough or the amount of leukemic stem cells is small enough, composite steady states are linearly stable. This result might give qualitative hints for therapeutic approaches.

Analysis of the model results in different scenarios of leukemic cell growth, among which the enhanced proliferation of malignant cells is the most prominent one. Nevertheless different scenarios are possible, such as induction of apoptosis or enhanced self-renewal. Enhanced self-renewal can partly compensate for reduced proliferation rates of malignant cells compared to healthy cells and vice versa. The case of reduced proliferation and enhanced self-renewal is of major biological interest, since it gives a putative explanation for intrinsic resistance of some cancers to cytotoxic treatment. Myelodysplastic syndromes are one example of this scenario [16, 26, 37, 19, 36]. Enhancement of self-renewal allows to establish a population of leukemic stem cells dividing slower than healthy stem cells and being therefore protected from cytotoxic agents. This also explains why dose acceleration of decitabine from the hypomethylating to the cytotoxic mode of action leads to a reduced outcome $[16,36]$, especially since hypomethylation seems to interfere with self-renewal [13].

The proposed model provides a rigorous framework for a systematic investigation of different scenarios of cancer initiation and to give putative answers to questions posed in the field of clinical applications. Diversity of scenarios described by the proposed model reflects the clinical hetero- 
geneity between different types of leukemias. This is a first step to respond to the need to develop specific treatment strategies dependent on the properties of malignant cells.

\section{Acknowledgements}

This work is a part of the Collaborative Research Center (SFB 873) "Maintenance and Differentiation of Stem Cells in Development and Disease". TS is a graduate student of the SFB 873. AM-C was supported by ERC Starting Grant "Biostruct" and Emmy Noether Programme of German Research Council (DFG). The authors are also thankful to the WIN Kolleg of Heidelberg Academy of Sciences and Humanities.

\section{References}

[1] J. Ablain, H. de The. Revisiting the differentiation paradigm in acute promyelocytic leukemia. Blood, 117 (2008), No. 22, 5795-5802.

[2] M. Adimy, F. Crauste. Modeling and asymptotic stability of a growth factor-dependent stem cell dynamics model with distributed delay. Discrete Contin. Dyn. Syst. Ser. B, 8 (2007), No. $1,19-38$.

[3] M. Adimy, F. Crauste, S. Ruan. A mathematical study of the hematopoiesis process with applications to chronic myelogenous leukemia. SIAM J. Appl. Math., 65 (2005), No. 4, 13281352.

[4] M. Adimy, F. Crauste, S. Ruan. Modelling hematopoiesis mediated by growth factors with applications to periodic hematological diseases. Bull. Math. Biol., 68 (2006), No. 8, 23212351.

[5] M. Aglietta, W. Piacibello, F. Sanavio, A. Stacchini, F. Apra, M. Schena, C. Mossetti, F. Carnino, F. Caligaris-Cappio, F. Gavosto. Kinetics of human hemopoietic cells after in vivo administration of granulocyte-macrophage colony-stimulating factor. J. Clin. Invest., 83 (1989), No. 2, 551-557.

[6] T. Alarcon, P. Getto, A. Marciniak-Czochra, MdM. Vivanco. A model for stem cell population dynamics with regulated maturation delay. Discr. Cont. Dyn. Systems B (2011), to appear.

[7] L. Andrey. Chaos in cancer. Med. Hypotheses, 28 (1989), No. 3, 143-144.

[8] O. Arino, M. Kimmel. Stability analysis of models of cell production systems. Math. Modelling, 7 (1986), No. 9-12, 1269-1300.

[9] M. Baum, MA. Chaplain, AR. Anderson, M. Douek, JS. Vaidya. Does breast cancer exist in a state of chaos? Eur. J. Cancer, 35 (1999), No. 6, 886-891. 
[10] R. Bejar, R. Levine, and BL. Ebert. Unraveling the molecular pathophysiology of myelodysplastic syndromes. J. Clin. Oncol., 29 (2011), No. 5, 504-514.

[11] J. Belair, MC. Mackey, JM. Mahaffy. Age-structured and two-delay models for erythropoiesis. Math. Biosci., 128 (1995), No. 1-2, 317-346.

[12] C. Bellan, L. Stefano, dF. Giulia, EA. Rogena, L. Lorenzo. Burkitt lymphoma versus diffuse large B-cell lymphoma: a practical approach. Hematol. Oncol., 28 (2010), No. 2, 53-56.

[13] MT. Bocker, I. Hellwig, A. Breiling, V. Eckstein, AD. Ho, F. Lyko. Genome-wide promoter DNA methylation dynamics of human hematopoietic progenitor cells during differentiation and aging. Blood, 117 (2011), No. 19: e182-e189.

[14] V. Bogner, L. Keil, KG. Kanz, C. Kirchhoff, BA. Leidel, W. Mutschler, P. Biberthaler. Very early posttraumatic serum alterations are signicantly associated to initial massive RBC substitution, injury severity, multiple organ failure and adverse clinical outcome in multiple injured patients. Eur. J. Med. Res., 14 (2009), No. 7, 284-291.

[15] D. Bonnet, JE. Dick. Human acute myeloid leukemia is organized as a hierarchy that originates from a primitive hematopoietic cell. Nat. Med., 3 (1997), No. 7, 730-737.

[16] J. Bryan, E. Jabbour, H. Prescott, G. Garcia-Manero, JP. Issa, H. Kantarjian. Current and future management options for myelodysplastic syndromes. Drugs, 70 (2010), No. 11, 13811394.

[17] EC. Buss, AD. Ho. Leukemia stem cells. Int. J. Cancer., 129 (2011), No. 10, 2328-2336.

[18] SY. Chen, YC. Huang, SP Liu, FJ Tsai, WC Shyu, SZ Lin. An overview of concepts for cancer stem cells. Cell Transplant., 20 (2011), No. 1, 113-120.

[19] BD. Cheson. Standard and low-dose chemotherapy for the treatment of myelodysplastic syndromes. Leuk. Res., Suppl. 1 (1998), S17-S21.

[20] H. Clevers. The cancer stem cell: premises, promises and challenges. Nat Med., 17 (2011), No. 3, 313-319.

[21] DS. Coffey. Self-organization, complexity and chaos: the new biology for medicine. Nat. Med., 4 (1998), No. 8, 882-885.

[22] C. Colijn, MC. Mackey. A mathematical model of hematopoiesis -I. periodic chronic myelogenous leukemia. J. Theor. Biol., 237 (2005), No. 2, 117-132.

[23] D. Dingli, JM. Pacheco. Stochastic dynamics and the evolution of mutations in stem cells. BMC Biol., 9:41 (2011).

[24] M. Doumic-Jauffret, PS. Kim, B. Perthame. Stability analysis of a simplied yet complete model for chronic myelogenous leukemia. Bull. Math. Biol., 72 (2010), No. 7, 1732-1759. 
[25] M. Doumic-Jauffret, A. Marciniak-Czochra, B. Perthame, JP. Zubelli. A Structured Population Model of Cell Differentiation. SIAM J. Appl. Math., 71 (2011), 1918-1940.

[26] P. Fenaux, GJ. Mufti, E. Hellstrom-Lindberg, V. Santini, C. Finelli, A. Giagounidis, R. Schoch, N. Gattermann, G. Sanz, A. List, SD. Gore, JF. Seymour, JM. Bennett, J. Byrd, J. Backstrom, L. Zimmerman, D. McKenzie, C. Beach, LR .Silverman; International Vidaza High-Risk MDS Survival Study Group. Efficacy of azacitidine compared with that of conventional care regimens in the treatment of higher-risk myelodysplastic syndromes: a randomised, open-label, phase III study. Lancet Oncol., 10 (2009), No. 3, 223-232.

[27] C. Foley, S. Bernard, MC. Mackey. Cost-effective G-CSF therapy strategies for cyclical neutropenia: Mathematical modelling based hypotheses. J. Theor. Biol., 238 (2006), No. 4, 754 763.

[28] W. Fried. Erythropoietin and erythropoiesis. Exp. Hematol., 37 (2009), No. 9, 1007-1015.

[29] FR. Gantmacher. The theory of matrices 2. Chelsea Publishing, New York, 1964.

[30] P. Getto, A. Marciniak-Czochra, Y. Nakata, MdM. Vivanco. Global dynamics of two compartment models for cell production systems with regulatory mechanisms. (2011), submitted.

[31] J. Guckenheimer, P. Holmes. Nonlinear Oscillations, Dynamical Systems, and Bifurcations of Vector Fields. Springer, New York, 2002.

[32] H. Haeno, RL. Levine, DG. Gilliland, F. Michor. A progenitor cell origin of myeloid malignancies., PNAS, 106 (2009),No. 39, 16616-16621.

[33] KJ. Hope, L. Jin, JE. Dick. Acute myeloid leukemia originates from a hierarchy of leukemic stem cell classes that differ in self-renewal capacity. Nat. Immun., 5 (2004), No. 7, 738-743.

[34] JH. Jandl (ed), Textbook of Hematology, Little Brown, Boston, MA, 1996.

[35] S. Janz, M. Potter, CS. Rabkin. Lymphoma- and leukemia-associated chromosomal translocations in healthy individuals. Genes Chromosomes Cancer, 36 (2003), No. 3, 211-223.

[36] H. Kantarjian, Y. Oki, G. Garcia-Manero, X. Huang, S. OBrien, J. Cortes, S. Faderl, C. Bueso-Ramos, F. Ravandi, Z. Estrov, A. Ferrajoli, W. Wierda, J. Shan, J. Davis, F. Giles, HI. Saba, JP. Issa. Results of a randomized study of 3 schedules of low-dose decitabine in higher-risk myelodysplastic syndrome and chronic myelomonocytic leukemia. Blood, 109 (2007), No. 1, 52-57.

[37] S. Knipp, B. Hildebrand, A. Kündgen, A. Giagounidis, G. Kobbe, R. Haas, C. Aul, N. Gattermann, U. Germing. Intensive chemotherapy is not recommended for patients aged $>60$ years who have myelodysplastic syndromes or acute myeloid leukemia with high-risk karyotypes. Cancer, 110 (2007), No. 2, 345-352. 
[38] N. Korde, SY. Kristinsson, O. Landgren. Monoclonal gammopathy of undetermined signicance (MGUS) and smoldering multiple myeloma (Smm): novel biological insights and development of early treatment strategies. Blood, 117 (2011), No. 21, 5573-5581.

[39] A. Lander, K. Gokoffski, F. Wan, Q. Nie, A. Calof. Cell lineages and the logic of proliferative control. PLoS biology, 7 (2009), No. 1, 84-100.

[40] SS. Lange, K. Takata, RD. Wood. DNA polymerases and cancer. Nat. Rev. Cancer, 11 (2011), No. 2, 96-110.

[41] PM. Lansdorp. Stem cell biology for the transfusionist. Vox Sang., 74 Suppl. 2 (1998), 91-94.

[42] JE. Layton, H. Hockman, WP. Sheridan, G. Morstyn. Evidence for a novel in vivo control mechanism of granulopoiesis: mature cell-related control of a regulatory growth factor. Blood, 74 (1989), No. 4, 1303-1307.

[43] W. Lo, C. Chou, K. Gokoffski, F. Wan, A. Lander, A. Calof, Q. Nie. Feedback regulation in multistage cell lineages. Math. Biosci. Eng., 6 (2009), No. 1, 59-82.

[44] MC. Mackey. Unified hypothesis for the origin of aplastic anemia and periodic hematopoiesis. Blood, 51 (1978), No. 5, 941-956.

[45] MC. Mackey, L. Glass, Oscillation and chaos in physiological control systems. Science, 197 (1977), No. 4300, 287-289.

[46] A. Marciniak-Czochra, T. Stiehl. Mathematical models of hematopoietic reconstitution after stem cell transplantation. In HG. Bock, T. Carraro, W. Jäger, S. Koerkel, R. Rannacher, JP. Schloeder (eds), Model Based Parameter Estimation: Theory and Applications. Springer, Heidelberg, 2011.

[47] A. Marciniak-Czochra, T. Stiehl, W. Jäger, AD. Ho, W. Wagner. Modeling of asymmetric cell division in hematopoietic stem cells - regulation of self-renewal is essential for efficient repopulation. Stem Cells Dev., 18 (2009), No. 3, 377-385.

[48] A. Marciniak-Czochra, T. Stiehl, W. Wagner. Modeling of replicative senescence in hematopoietic development. Aging (Albany NY), 1 (2009), No. 8, 723-732.

[49] D. Metcalf. Hematopoietic cytokines. Blood, 111 (2008), No. 2, 485-491.

[50] F. Michor, TP. Hughes, Y. Iwasa, S. Branford, NP. Shah, CL. Sawyers, MA. Nowak. Dynamics of chronic myeloid leukaemia. Nature, 435 (2005), No. 7046, 1267-1270.

[51] KA. Moore, IR. Lemischka. Stem cells and their niches. Science, 311 (2006), No. 5769, 1880-1805.

[52] D. Morgan, A. Murray, T. Hunt, P. Nurse. In: Alberts Molecular Biology of the Cell, 4th Edition, Garland Science, New York, 2002. 
[53] I. Munk Pedersen, J. Reed. Microenvironmental interactions and survival of CLL B-cells. Leuk. Lymphoma, 45 (2004), No. 12, 2365-2372.

[54] Y. Nakata, P. Getto, A. Marciniak-Czochra, T. Alarcon. Stability analysis of multi-compartment models for cell production systems. J. Biol. Dyn., (2011), doi: 10.1080/17513758.2011.558214.

[55] L. Pujo-Menjouet, S. Bernard, MC. Mackey. Long period oscillations in a G0 model of hematopoietic stem cells. SIAM J. Appl. Dyn. Syst, 4 (2005), No. 2, 312-332.

[56] T. Reya, SJ. Morrison, MF. Clarke, IL. Weissman. Stem cells, cancer, and cancer stem cells. Nature, 414 (2001), No. 6859, 105-111.

[57] I. Roeder, M. Herberg, M. Horn. An age-structured model of hematopoietic stem cell organization with application to chronic myeloid leukemia. Bull. Math. Biol., 71 (2009), No. 3, 602-626.

[58] I. Roeder, M. Horn, I. Glauche, A. Hochhaus, MC. Mueller, M. Loeffler. Dynamic modeling of imatinib-treated chronic myeloid leukemia: functional insights and clinical implications. Nat. Med., 12 (2006), No. 10, 1181-1184.

[59] I. Roeder, M. Loeffler. A novel dynamic model of hematopoietic stem cell organization based on the concept of within-tissue plasticity. Exp. Hematol., 30 (2002), 853-861.

[60] R. Rudnicki. Chaoticity of the blood cell production system. Chaos, doi: 10.1063/1.3258364, 2009.

[61] F. Schueler, C. Hirt, G. Doelken. Chromosomal translocation t $(14 ; 18)$ in healthy individuals. Semin. Cancer. Biol., 13 (2003), No. 3, 203-209.

[62] K. Shinjo, A. Takeshita, K. Ohnishi, R. Ohno. Granulocyte colony-stimulating factor receptor at various stages of normal and leukemic hematopoietic cells. Leuk. Lymphoma, 25 (1997), No. 1-2, 37-46.

[63] S. Soltanian, MM. Matin. Cancer stem cells and cancer therapy. Tumour Biol., 32 (2011), No. 3, 425-440.

[64] T. Stiehl, A. Marciniak-Czochra. Characterization of stem cells using mathematical models of multistage cell lineages. Mathematical and Computer Modelling, 53 (2011), No. 7-8, 15051517.

[65] JE. Till, L. Siminovitch, EA. McCulloch. Stochastic Model of Stem Cell Proliferation Based on Growth of Spleen Colony-Forming Cells. PNAS, 51 (1964), 29-49.

[66] C. Tomasetti, D. Levy. Role of symmetric and asymmetric division of stem cells in developing drug resistance. PNAS., 107 (2010), No. 39. 16766-16771. 
[67] M. Tormo, I. Marugan, M. Calabuig. Myelodysplastic syndromes: an update on molecular pathology. Clin. Transl. Oncol., 12 (2010), No. 10, 652-661.

\section{A Proof of Proposition 24}

- The linearization $L$ around the composite steady $\left(c_{1}^{\star}, c_{2}^{\star}, l_{1}^{\star}, l_{2}^{\star}\right)$ state is given by

$$
L:=\left(\begin{array}{cccc}
0 & -\frac{k p_{1}^{c}}{2 a_{1}^{c}} c_{1}^{\star} & 0 & -\frac{k p_{1}^{c}}{2 a_{1}^{c}} c_{1}^{\star} \\
p_{1}^{c} & \frac{k p_{1}^{c}}{2 a_{1}^{c}} c_{1}^{\star}-d_{2}^{c} & 0 & \frac{k p_{1}^{1}}{2 a_{1}^{c}} c_{1}^{\star} \\
0 & -\frac{k p_{1}^{l}}{2 a_{1}^{l}} l_{1}^{\star} & 0 & -\frac{k p_{1}^{l}}{2 a_{1}^{l}} l_{1}^{\star} \\
0 & \frac{k p_{1}^{l}}{2 a_{1}^{l}} l_{1}^{\star} & p_{1}^{l} & \frac{k p_{1}^{l}}{2 a_{1}^{l}} l_{1}^{\star}-d_{2}^{l}
\end{array}\right) .
$$

This follows using $s^{\star}=\frac{1}{2 a_{1}^{l}}=\frac{1}{2 a_{1}^{c}}, \frac{\partial}{\partial c_{2}} s=\frac{\partial}{\partial l_{2}} s=-k s^{2}$.

- Let $v:=\left(-\frac{d_{2}^{c}}{p_{1}^{c}},-1, \frac{d_{2}^{l}}{p_{1}^{l}}, 1\right)^{T}$, then

$$
L \cdot v=0
$$

- The center manifold of the equilibrium point $\left(c_{1}^{\star}, c_{2}^{\star}, l_{1}^{\star}, l_{2}^{\star}\right)$ is invariant under the flow of the system and is tangent to the center eigenvector in the equilibrium point, c.f. Section 3.2 of ref. [31].

Due to Proposition 7 the manifold of steady states is given as:

$$
\left(\begin{array}{c}
c_{1} \\
c_{2} \\
l_{1} \\
l_{2}
\end{array}\right)=\left(\begin{array}{c}
\frac{d_{2}^{c}}{p_{1}^{c}}\left(\frac{2 a_{1}^{c}-1}{k}-l_{2}\right) \\
\frac{2 a_{1}^{c}-1}{k}-l_{2} \\
\frac{d_{2}^{l}}{p_{1}^{l}} l_{2} \\
l_{2}
\end{array}\right)=\left(\begin{array}{c}
\frac{d_{2}^{c}}{p_{1}^{c}} \frac{2 a_{1}^{c}-1}{k} \\
\frac{2 a_{1}^{c}-1}{k} \\
0 \\
0
\end{array}\right)+l_{2}\left(\begin{array}{c}
-\frac{d_{2}^{c}}{p_{1}^{c}} \\
-1 \\
\frac{d_{2}^{l}}{p_{1}^{l}} \\
1
\end{array}\right)
$$

Therefore $v$ is parallel to the manifold of steady states. Since $\left(c_{1}^{\star}, c_{2}^{\star}, l_{1}^{\star}, l_{2}^{\star}\right)$ lies on the manifold of steady states, it is tangent to $v$ in $\left(c_{1}^{\star}, c_{2}^{\star}, l_{1}^{\star}, l_{2}^{\star}\right)$. Since on each point on the manifold of steady states the system is in an equilibrium the manifold is invariant under the flow of the system. Therefore the manifold of steady states is a center manifold in the case where only one eigenvalue has zero real part.

\section{B Proof of Lemma 25}

We use notations from equation (3.1).

(i) The zero order term of $\tilde{\chi}_{M}$ is equivalent to $\nu^{c} \alpha^{c} \cdot d_{2}^{l}+\nu^{l} \alpha^{l} \cdot d_{2}^{c}$ and therefore positive, since 
$d_{2}^{c}>0, d_{2}^{l}>0, \nu^{c} \alpha^{c}>0$, and $\nu^{l} \alpha^{l}>0$.

(ii) Exploiting

$$
\begin{aligned}
\bar{s} & =\frac{d_{1}^{c}+p_{1}^{c}}{2 a_{1}^{c} p_{1}^{c}}=\frac{d_{1}^{l}+p_{1}^{l}}{2 a_{1}^{l} p_{1}^{l}}, \\
\bar{c}_{1} & =\frac{\bar{c}_{2} d_{2}^{c}}{2\left(1-a_{1}^{c} \bar{s}\right) p_{1}^{c}}, \\
\bar{l}_{1} & =\frac{\bar{l}_{2} d_{2}^{l}}{2\left(1-a_{1}^{l} \bar{s}\right) p_{1}^{l}} \text { and } \\
k \bar{c}_{2} & =\frac{1}{\bar{s}}-1-k \bar{l}_{2},
\end{aligned}
$$

it follows for the second order term:

$$
-\left(\rho^{l}+\rho^{c}\right)=\left(-2 \frac{d_{2}^{c} a_{1}^{c} p_{1}^{c}}{p_{1}^{c}-d_{1}^{c}}+\frac{d_{2}^{c}\left(p_{1}^{c}+d_{1}^{c}\right)}{p_{1}^{c}-d_{1}^{c}}+\frac{\left(p_{1}^{c}+d_{1}^{c}\right) d_{2}^{c}}{p_{1}^{c}-d_{1}^{c}} k \bar{l}_{2}-\frac{d_{2}^{l}\left(d_{1}^{l}+p_{1}^{l}\right)}{p_{1}^{l}-d_{1}^{l}} k \bar{l}_{2}\right) s+d_{2}^{c}+d_{2}^{l} .
$$

We define

$$
\begin{aligned}
\eta & :=\left(-2 \frac{d_{2}^{c} a_{1}^{c} p_{1}^{c}}{p_{1}^{c}-d_{1}^{c}}+\frac{d_{2}^{c}\left(p_{1}^{c}+d_{1}^{c}\right)}{p_{1}^{c}-d_{1}^{c}}\right) s+d_{2}^{c}, \\
\xi & :=-\frac{d_{2}^{l}\left(d_{1}^{l}+p_{1}^{l}\right)}{p_{1}^{l}-d_{1}^{l}} k \bar{l}_{2} s+d_{2}^{l}, \\
\theta & :=\frac{\left(p_{1}^{c}+d_{1}^{c}\right) d_{2}^{c}}{p_{1}^{c}-d_{1}^{c}} k \bar{l}_{2} s \geq 0,
\end{aligned}
$$

with $-\left(\rho^{l}+\rho^{c}\right)=\eta+\xi+\theta$.

It calculates:

$$
\begin{aligned}
\eta & =\frac{d_{2}^{c}}{2\left(p_{1}^{c}-d_{1}^{c}\right) a_{1}^{c} p_{1}^{c}}\left(-4 p_{1}^{c} a_{1}^{c} d_{1}^{c}+\left(d_{1}^{c}\right)^{2}+2 d_{1}^{c} p_{1}^{c}+\left(p_{1}^{c}\right)^{2}\right) \\
& >\frac{d_{2}^{c}}{2\left(p_{1}^{c}-d_{1}^{c}\right) a_{1}^{c} p_{1}^{c}}\left(\left(d_{1}^{c}\right)^{2}-2 d_{1}^{c} p_{1}^{c}+\left(p_{1}^{c}\right)^{2}\right) \\
& =\frac{d_{2}^{c}}{2\left(p_{1}^{c}-d_{1}^{c}\right) a_{1}^{c} p_{1}^{c}}\left(d_{1}^{c}-p_{1}^{c}\right)^{2} \geq 0,
\end{aligned}
$$

since $a_{1}^{c}<1$ and $p_{1}^{c}-d_{1}^{c}>\left(2 a_{1}^{c}-1\right) p_{1}^{c}-d_{1}^{c}$, which is assumed to be positive, if we are interested in nonnegative steady states (Proposition 10). Since we assume $\bar{l}_{2} \geq 0$ and $\bar{c}_{2} \geq 0, k \bar{c}_{2}+k \bar{l}_{2}=\frac{1}{s}-1$ 
implies $k \bar{l}_{2} \leq \frac{1}{s}-1=\frac{2 a_{1}^{l} p_{1}^{l}-d_{1}^{l}-p_{1}^{l}}{p_{1}^{l}+d_{1}^{l}}$. It holds

$$
\begin{aligned}
\xi & \geq-\frac{d_{2}^{l}\left(d_{1}^{l}+p_{1}^{l}\right)}{p_{1}^{l}-d_{1}^{l}} \frac{2 a_{1}^{l} p_{1}^{l}-d_{1}^{l}-p_{1}^{l}}{p_{1}^{l}+d_{1}^{l}} s+d_{2}^{l} \\
& =\frac{d_{2}^{l}}{2\left(p_{1}^{l}-d_{1}^{l}\right) a_{1}^{l} p_{1}^{l}}\left(-4 p_{1}^{l} a_{1}^{l} d_{1}^{l}+d_{1}^{l}{ }^{2}+2 d_{1}^{l} p_{1}^{l}+p_{1}^{l}{ }^{2}\right) \\
& >\frac{d_{2}^{l}}{2\left(p_{1}^{l}-d_{1}^{l}\right) a_{1}^{l} p_{1}^{l}}\left(p_{1}^{l}-d_{1}^{l}\right)^{2} \geq 0 .
\end{aligned}
$$

Consequently the second order term is positive.

(iii) follows by direct calculation.

\section{Proof of Lemma 27}

We write $\tilde{\beta}=\rho^{l}+\zeta$, with

$$
\begin{aligned}
& \rho^{l}:=\left(p_{1}^{l}\left(d_{2}^{l}\right)^{2}+\left(d_{2}^{c}\right)^{2} d_{2}^{l}\right) \bar{s}^{2}-\hat{\alpha} \bar{s}(1-\bar{s})>0, \\
& \zeta:=-\left(p_{1}^{c}\left(d_{2}^{c}\right)^{2}+d_{2}^{c}\left(d_{2}^{l}\right)^{2}\right) \bar{s}^{2}<0 .
\end{aligned}
$$

Exploiting $X=k \bar{l}_{2}<2 a_{1}^{c}-1$ and $a_{1}^{c}<1$ it follows

$$
\begin{aligned}
H_{2}(X) & =\hat{\alpha} \bar{s}^{2} X^{2}+\rho^{l} X+\zeta X+\tilde{\gamma} \\
& \geq \hat{\alpha} \bar{s}^{2} X^{2}+\rho^{l} X+\zeta\left(2 a_{1}^{c}-1\right)+\tilde{\gamma} \\
& =\hat{\alpha} \bar{s}^{2} X^{2}+\rho^{l} X+\left(1-\left(2 a_{1}^{c}-1\right) s\right) \bar{s} d_{2}^{c}\left(d_{2}^{l}\right)^{2}+\left(d_{2}^{c}\right)^{2} d_{2}^{l} \bar{s}^{2} \\
& >\hat{\alpha} \bar{s}^{2} X^{2}+\rho^{l} X \\
& \geq \hat{\alpha} \bar{s}^{2} X^{2}-\hat{\alpha} \bar{s}(1-\bar{s}) X \\
& =\hat{\alpha} \bar{s} X(\bar{s} X-(1-\bar{s}))
\end{aligned}
$$

which is positive in the relevant range $X \in\left[0, \frac{1}{\bar{s}}-1=2 a_{1}^{c}-1\right]$.

\section{Proof of Lemma 29}

We write $\tilde{\beta}=\rho^{l}+\zeta$, with

$$
\begin{aligned}
& \rho^{l}:=\left(p_{1}^{l}\left(d_{2}^{l}\right)^{2}+\left(d_{2}^{c}\right)^{2} d_{2}^{l}\right) \bar{s}^{2}>0 \\
& \zeta:=-\left(p_{1}^{c}\left(d_{2}^{c}\right)^{2}+d_{2}^{c}\left(d_{2}^{l}\right)^{2}\right) \bar{s}^{2}<0
\end{aligned}
$$


Exploiting $X<2 a_{1}^{c}-1$ and $a_{1}^{c}<1$ it follows

$$
\begin{aligned}
H_{2}(X) & =\rho^{l} X+\zeta X+\tilde{\gamma} \\
& \geq \rho^{l} X+\zeta\left(2 a_{1}^{c}-1\right)+\tilde{\gamma} \\
& =\rho^{l} X+\left(1-\left(2 a_{1}^{c}-1\right) \bar{s}\right) \bar{s} d_{2}^{c}\left(d_{2}^{l}\right)^{2}+\left(d_{2}^{c}\right)^{2} d_{2}^{l} \bar{s}^{2} \\
& >\rho^{l} X \geq 0 .
\end{aligned}
$$

\section{E Proof of Proposition 31}

Remark 35. If $\tilde{\beta} \geq 0$, then $H_{2}(X)>0$, since $\tilde{\gamma}>0$.

Corollary 36. Assume $k^{l}=k^{c}$ and $d_{1}^{c}=d_{1}^{l}=0$. Then it is necessary that $\hat{\alpha}>0$ and $\tilde{\beta}<0$ to obtain $\mathrm{H}_{2}<0$ within the biologically relevant range of parameters.

Proof: The corollary follows from Propositions 27 and 29 and from Remark 35.

We now analyze if there exist relevant parameters that fulfill $H_{2}<0$. Note that $H_{2}>0$ implies linear stability due to the Hurwitz theorem, since the quadratic and absolute term of $\tilde{\chi}_{M}(\lambda)$ are positive (Lemma 25).

Remark 37. $\hat{\alpha}>0$ implies

(i) $\left(p_{1}^{l} d_{2}^{l}-p_{1}^{c} d_{2}^{c}\right)>0$ and $\left(d_{2}^{c}-d_{2}^{l}\right)>0$ or

(ii) $\left(p_{1}^{l} d_{2}^{l}-p_{1}^{c} d_{2}^{c}\right)<0$ and $\left(d_{2}^{c}-d_{2}^{l}\right)<0$.

Renaming variables $\left(p_{i}^{c} \leftrightarrow p_{i}^{l}, a_{i}^{c} \leftrightarrow a_{i}^{l}, c_{i} \leftrightarrow l_{i}^{l}, d_{2}^{l} \leftrightarrow d_{2}^{c}\right)$ does not change the ODE system, but transforms case (i) to case (ii). For this reason we can assume without loss of generality that $\left(p_{1}^{l} d_{2}^{l}-p_{1}^{c} d_{2}^{c}\right)>0$ and $\left(d_{2}^{c}-d_{2}^{l}\right)>0$.

Remark 38. For the remainder of this Section we assume

- $\left(p_{1}^{l} d_{2}^{l}-p_{1}^{c} d_{2}^{c}\right)>0$,

- $\left(d_{2}^{c}-d_{2}^{l}\right)>0$,

- $k^{l}=k^{c}$ and

- $d_{1}^{c}=d_{1}^{l}=0$.

In the following we set $\tilde{\delta}:=\left[p_{1}^{l}\left(d_{2}^{l}\right)^{2}-p_{1}^{c}\left(d_{2}^{c}\right)^{2}+d_{2}^{c} d_{2}^{l}\left(d_{2}^{c}-d_{2}^{l}\right)\right]$, then $\tilde{\beta}=\bar{s}^{2} \tilde{\delta}-\hat{\alpha} \bar{s}(1-\bar{s})$.

Lemma 39. Let $\tilde{\beta}<0$. We consider $H_{2}(X)$ as a quadratic polynomial in $X=k \bar{l}_{2}$. The minimal point of the corresponding parabola lies in the relevant range $0 \leq k \bar{l}_{2} \leq\left(2 a_{1}^{c}-1\right)$ if and only if

$$
\hat{\alpha}(1-\bar{s})+\tilde{\delta} \bar{s} \geq 0
$$


Proof: The minimum of this parabola is at $X_{\min }:=-\frac{\tilde{\beta}}{2 \hat{\alpha} \bar{s}^{2}}$. Since we assumed $\tilde{\beta}<0$, it follows that $X_{\min }>0$. It holds

$$
\begin{aligned}
-\frac{\tilde{\beta}}{2 \hat{\alpha} \bar{s}^{2}} & \leq \frac{1}{\bar{s}}-1 \Leftrightarrow \\
-\tilde{\beta} & \leq 2(1-\bar{s}) \bar{s} \hat{\alpha} \quad \Leftrightarrow \\
-\bar{s}^{2} \tilde{\delta}+\hat{\alpha} \bar{s}(1-\bar{s}) & \leq 2(1-\bar{s}) \bar{s} \hat{\alpha} \quad \Leftrightarrow \\
\hat{\alpha}(1-\bar{s})+\tilde{\delta} \bar{s} & \geq 0 .
\end{aligned}
$$

\section{Lemma 40.}

(i) Let $\tilde{\beta}<0$. Assume $\hat{\alpha}(1-\bar{s})+\tilde{\delta} \bar{s} \geq 0$. There exists $k l_{2}$ in the relevant range $\left[0,\left(2 a_{1}^{c}-1\right)\right]$ such that $H_{2}\left(k l_{2}\right)<0$ if and only if $\frac{-\tilde{\beta}^{2}}{4 \hat{\alpha} \bar{s}^{2}}+\tilde{\gamma}<0$.

(ii) If $\hat{\alpha}(1-\bar{s})+\tilde{\delta} \bar{s}<0$, then $H_{2}(X)>0$.

Proof: (i) Due to Lemma 39 the minimum of $H_{2}(X)$ lies within the relevant range. The minimum value of $H_{2}(X)$ is $\frac{-\tilde{\beta}^{2}}{4 \hat{\alpha} \bar{s}^{2}}+\tilde{\gamma} . " \Rightarrow$ "If the minimum is negative and lies within the relevant range then there exist relevant parameters such that $H_{2}(X)<0$. " $\Leftarrow$ " If there exist relevant parameters such that $\mathrm{H}_{2}<0$, then the minimum of $\mathrm{H}_{2}(X)$ in the relevant range is negative and therefore $\frac{-\tilde{\beta}^{2}}{4 \hat{\alpha} \bar{s}^{2}}+\tilde{\gamma}<0$.

(ii) Due to Lemma 39 and since $X_{\text {min }}>0, X_{\min }$ is greater than the maximal relevant value of $k \bar{l}_{2}$. Therefore the minimum of the parabola $H_{2}(X)$ in the relevant range is at $X=\frac{1}{\bar{s}}-1$. It holds

$$
\begin{aligned}
H_{2}\left(\frac{1}{\bar{s}}-1\right)= & \hat{\alpha}(1-\bar{s})^{2}+\tilde{\beta}\left(\frac{1}{\bar{s}}-1\right)+\tilde{\gamma} \\
= & \hat{\alpha}(1-\bar{s})^{2}+\tilde{\delta} \bar{s}(1-\bar{s})-\hat{\alpha}(1-\bar{s})^{2}+\tilde{\gamma} \\
= & {\left[p_{1}^{l}\left(d_{2}^{l}\right)^{2}-p_{1}^{c}\left(d_{2}\right)^{2}+d_{2} d_{2}^{l}\left(d_{2}^{c}-d_{2}^{l}\right)\right] \bar{s}(1-\bar{s})+d_{2}^{c}\left(d_{2}^{l}\right)^{2} \bar{s}+\left(d_{2}^{c}\right)^{2} d_{2}^{l} \bar{s}^{2} } \\
& +\left(d_{2}^{c}\right)^{2} p_{1}^{c}\left(2 a_{1}^{c}-1\right) \bar{s}^{2} \\
= & {\left[p_{1}^{l}\left(d_{2}^{l}\right)^{2}-p_{1}^{c}\left(d_{2}^{c}\right)^{2}+d_{2} d_{2}^{l}\left(d_{2}^{c}-d_{2}^{l}\right)\right] \bar{s}(1-\bar{s})+d_{2}^{c}\left(d_{2}^{l}\right)^{2} \bar{s}+\left(d_{2}^{c}\right)^{2} d_{2}^{l} \bar{s}^{2} } \\
& +\left(d_{2}^{c}\right)^{2} p_{1}^{c}(1-\bar{s}) \bar{s} \\
= & {\left[p_{1}^{l}\left(d_{2}^{l}\right)^{2}+d_{2}^{c} d_{2}^{l}\left(d_{2}^{c}-d_{2}^{l}\right)\right] \bar{s}(1-\bar{s})+d_{2}^{c}\left(d_{2}^{l}\right)^{2} \bar{s}+\left(d_{2}^{c}\right)^{2} d_{2}^{l} \bar{s}^{2}>0 . }
\end{aligned}
$$

Corollary 41. (Reformulation of Corollary 36) Assume $k^{l}=k^{c}$ and $d_{1}^{c}=d_{1}^{l}=0$. For existence of $k \bar{l}_{2}$ in the biological relevant range with $H_{2}\left(k \bar{l}_{2}\right)<0$ it is necessary and sufficient that $\hat{\alpha}>0$, $\tilde{\beta}<0, \hat{\alpha}(1-\bar{s})+\tilde{\delta} \bar{s} \geq 0$ and $\frac{-\tilde{\beta}^{2}}{4 \hat{\alpha} \bar{s}^{2}}+\tilde{\gamma}<0$.

Proof: The corollary follows from Propositions 27, 29, Lemmas 39, 40 and and from Remark 35.

We now elaborate on this case and try to give a more explicit formulation. 
Lemma 42. Assume $\hat{\alpha}(1-\bar{s})+\tilde{\delta} \bar{s} \geq 0$.

(i) It holds $\tilde{\beta}<0$ if and only if $0<\bar{s}<\frac{\hat{\alpha}}{\hat{\alpha}+\tilde{\delta}}$.

(ii) There exists $\bar{s} \in\left(\frac{1}{2}, 1\right)$ with $\tilde{\beta}<0$ if and only if $p_{1}^{c}>\frac{p_{1}^{l}\left(2 d_{2}^{l}-d_{2}^{c}\right)+\left(d_{2}^{c}\right)^{2}-d_{2}^{c} d_{2}^{l}}{d_{2}^{c}}$.

Proof: (i) Note that $\hat{\alpha}(1-\bar{s})+\tilde{\delta} \bar{s} \geq 0, \hat{\alpha}>0$, implies $\hat{\alpha}+\tilde{\delta}>0$. It holds $\tilde{\beta}=\bar{s}((\hat{\alpha}+\tilde{\delta}) \bar{s}-\hat{\alpha})$, which gives the result.

(ii) Due to (i) $\tilde{\beta}<0$ if and only if $0<\bar{s}<\frac{\hat{\alpha}}{\hat{\alpha}+\tilde{\delta}}$. It is therefore necessary and sufficient for existence of $\bar{s}>0.5$ that $\frac{\hat{\alpha}}{\hat{\alpha}+\tilde{\delta}}>\frac{1}{2}$, which is equivalent to $\hat{\alpha}>\tilde{\delta}$, which is equivalent to $p_{1}^{c}>$ $\frac{p_{1}^{l}\left(2 d_{2}^{l}-d_{2}^{c}\right)+\left(d_{2}^{c}\right)^{2}-d_{2}^{c} d_{2}^{l}}{d_{2}^{c}}$ :

$$
\begin{aligned}
\left(p_{1}^{l} d_{2}^{l}-p_{1}^{c} d_{2}^{c}\right)\left(d_{2}^{c}-d_{2}^{l}\right) & >p_{1}^{l}\left(d_{2}^{l}\right)^{2}-p_{1}\left(d_{2}^{c}\right)^{2}+d_{2}^{c} d_{2}^{l}\left(d_{2}^{c}-d_{2}^{l}\right) \Leftrightarrow \\
p_{1}^{c} d_{2}^{c} & >2 p_{1}^{l} d_{2}^{l}-p_{1}^{l} d_{2}^{c}+d_{2}^{c}\left(d_{2}^{c}-d_{2}^{l}\right) \Leftrightarrow \\
p_{1}^{c} & >\frac{p_{1}^{l}\left(2 d_{2}^{l}-d_{2}^{c}\right)+\left(d_{2}^{c}\right)^{2}-d_{2}^{c} d_{2}^{l}}{d_{2}^{c}} .
\end{aligned}
$$

With this result we can reformulate Corollary 41:

Corollary 43. (Reformulation of Corollary 41) Assume $k^{l}=k^{c}$ and $d_{1}^{c}=d_{1}^{l}=0$. After possible renaming of variables $\left(a_{i}^{c} \leftrightarrow a_{i}^{l}, p_{i}^{c} \leftrightarrow p_{i}^{l}, d_{2}^{l} \leftrightarrow d_{2}^{c}\right)$ it holds: For existence of $k \bar{l}_{2}$ in the biologically relevant range with $H_{2}\left(k \bar{l}_{2}\right)<0$ it is necessary and sufficient that $d_{2}^{c}>d_{2}^{l}, p_{1}^{l} d_{2}^{l}>p_{1}^{c} d_{2}^{c}, 0<\bar{s}<$ $\frac{\hat{\alpha}}{\hat{\alpha}+\tilde{\delta}}, p_{1}^{c}>\frac{p_{1}^{l}\left(2 d_{2}^{l}-d_{2}^{c}\right)+\left(d_{2}^{c}\right)^{2}-d_{2}^{c} d_{2}^{l}}{d_{2}^{c}}, \hat{\alpha}(1-\bar{s})+\tilde{\delta} \bar{s} \geq 0$ and $\frac{-\tilde{\beta}^{2}}{4 \hat{\alpha} \bar{s}^{2}}+\tilde{\gamma}<0$.

Proof: The corollary follows from Propositions 27, 29, Lemmas 39, 40 and 42 and from Remarks 35 and 37.

We further elaborate on the condition $\hat{\alpha}(1-\bar{s})+\tilde{\delta} \bar{s} \geq 0$ and on compatibility of $p_{1}^{c}>\frac{p_{1}^{l}\left(2 d_{2}^{l}-d_{2}^{c}\right)+\left(d_{2}^{c}\right)^{2}-d_{2}^{c} d_{2}^{l}}{d_{2}^{c}}$ and $p_{1}^{l} d_{2}^{l}>p_{1}^{c} d_{2}^{c}$.

Lemma 44. $\hat{\alpha}(1-\bar{s})+\tilde{\delta} \bar{s} \geq 0$ is equivalent to

$$
p_{1}^{c} \leq \frac{p_{1}^{l} d_{2}^{l}\left(d_{2}^{c}-d_{2}^{l}\right)(1-\bar{s})+d_{2}^{c} d_{2}^{l}\left(d_{2}^{c}-d_{2}^{l}\right) \bar{s}+p_{1}^{l}\left(d_{2}^{l}\right)^{2} \bar{s}}{d_{2}^{c}\left(d_{2}^{c}-d_{2}^{l}\right)(1-\bar{s})+\left(d_{2}^{c}\right)^{2} \bar{s}} .
$$

Proof: The proof follows from a direct calculation:

$$
\begin{aligned}
\hat{\alpha}(1-\bar{s})+\tilde{\delta} \bar{s} & \geq 0 \Leftrightarrow \\
\left(p_{1}^{l} d_{2}^{l}-p_{1}^{c} d_{2}^{c}\right)\left(d_{2}^{c}-d_{2}^{l}\right)(1-\bar{s})+\bar{s} p_{1}^{l}\left(d_{2}^{l}\right)^{2}-\bar{s} p_{1}^{c}\left(d_{2}^{c}\right)^{2}+d_{2}^{c} d_{2}^{l}\left(d_{2}^{c}-d_{2}^{l}\right) \bar{s} & \geq 0 \Leftrightarrow \\
\frac{p_{1}^{l} d_{2}^{l}\left(d_{2}^{c}-d_{2}^{l}\right)(1-\bar{s})+d_{2}^{c} d_{2}^{l}\left(d_{2}^{c}-d_{2}^{l}\right) \bar{s}+p_{1}^{l}\left(d_{2}^{l}\right)^{2} \bar{s}}{d_{2}^{c}\left(d_{2}^{c}-d_{2}^{l}\right)(1-\bar{s})+\left(d_{2}^{c}\right)^{2} \bar{s}} & \geq p_{1}^{c} .
\end{aligned}
$$


Since our choice of notation and $\hat{\alpha}>0$ requires $p_{1}^{l} d_{2}^{l}>p_{1}^{c} d_{2}^{c}$, it is necessary that $p_{1}^{l} \frac{d_{2}^{l}}{d_{2}^{c}}>$ $\frac{p_{1}^{l}\left(2 d_{2}^{l}-d_{2}^{c}\right)+\left(d_{2}^{c}\right)^{2}-d_{2}^{c} d_{2}^{l}}{d_{2}^{c}}$ to satisfy Lemma 42 .

Lemma 45. To fulfill $p_{1}^{l} \frac{d_{2}^{l}}{d_{2}^{c}}>\frac{p_{1}^{l}\left(2 d_{2}^{l}-d_{2}^{c}\right)+\left(d_{2}^{c}\right)^{2}-d_{2}^{c} d_{2}^{l}}{d_{2}^{c}}$ it is necessary and sufficient that $p_{1}^{l}>d_{2}^{c}$.

Proof: The proof follows from a direct calculation:

$$
\begin{aligned}
p_{1}^{l} d_{2}^{l} & >p_{1}^{l}\left(2 d_{2}^{l}-d_{2}^{c}\right)+\left(d_{2}^{c}\right)^{2}-d_{2}^{c} d_{2}^{l} \Leftrightarrow \\
p_{1}^{l}\left(d_{2}^{c}-d_{2}^{l}\right) & >d_{2}^{c}\left(d_{2}^{c}-d_{2}^{l}\right) .
\end{aligned}
$$

Remark 46 (Biological Interpretation). To destabilize an equilibrium of coexistence it is necessary that proliferation of leukemic stem cells is larger than death rate of mature healthy cells. This shows that the relation between properties of stem cells and mature cells is important to destabilize coexistence and that a certain amount of leukemic cell proliferation is necessary. By renaming of variables $\left(a_{i}^{c} \leftrightarrow a_{i}^{l}, p_{i}^{c} \leftrightarrow p_{i}^{l}\right.$, $d_{2}^{l} \leftrightarrow d_{2}^{c}$ ) we obtain the corresponding relation between proliferation of healthy cells and death rate of leukemic cells.

Lemma 47. If $p_{1}^{l}>d_{2}^{c}$ it holds:

$p_{1}^{l} \frac{d_{2}^{l}}{d_{2}^{c}}>\frac{p_{1}^{l} d_{2}^{l}\left(d_{2}^{c}-d_{2}^{l}\right)(1-\bar{s})+d_{2}^{c} d_{2}^{l}\left(d_{2}^{c}-d_{2}^{l}\right) \bar{s}+p_{1}^{l}\left(d_{2}^{l}\right)^{2} \bar{s}}{d_{2}^{c}\left(d_{2}^{c}-d_{2}^{l}\right)(1-\bar{s})+\left(d_{2}^{c}\right)^{2} \bar{s}}>\frac{p_{1}^{l}\left(2 d_{2}^{l}-d_{2}^{c}\right)+\left(d_{2}^{c}\right)^{2}-d_{2}^{c} d_{2}^{l}}{d_{2}^{c}}$.

Proof: The proof follows from a direct calculation:

$$
\begin{gathered}
p_{1}^{l} \frac{d_{2}^{l}}{d_{2}^{c}}>\frac{p_{1}^{l} d_{2}^{l}\left(d_{2}^{c}-d_{2}^{l}\right)(1-\bar{s})+d_{2}^{c} d_{2}^{l}\left(d_{2}^{c}-d_{2}^{l}\right) \bar{s}+p_{1}^{l}\left(d_{2}^{l}\right)^{2} \bar{s}}{d_{2}^{c}\left(d_{2}^{c}-d_{2}^{l}\right)(1-\bar{s})+\left(d_{2}^{c}\right)^{2} \bar{s}} \Leftrightarrow \\
p_{1}^{l}\left(d_{2}^{c}-d_{2}^{l}\right) d_{2}^{l}>d_{2}^{c} d_{2}^{l}\left(d_{2}^{c}-d_{2}^{l}\right), \\
\frac{p_{1}^{l} d_{2}^{l}\left(d_{2}^{c}-d_{2}^{l}\right)(1-\bar{s})+d_{2}^{c} d_{2}^{l}\left(d_{2}^{c}-d_{2}^{l}\right) \bar{s}+p_{1}^{l}\left(d_{2}^{l}\right)^{2} \bar{s}}{d_{2}^{c}\left(d_{2}^{c}-d_{2}^{l}\right)(1-\bar{s})+\left(d_{2}^{c}\right)^{2} \bar{s}}>\frac{p_{1}^{l}\left(2 d_{2}^{l}-d_{2}^{c}\right)+\left(d_{2}^{c}\right)^{2}-d_{2}^{c} d_{2}^{l}}{d_{2}^{c}} \Leftrightarrow \\
d_{2}^{c}\left(d_{2}^{c}-d_{2}^{l}\right)^{2}
\end{gathered}
$$

Remark 48. Lemma 47 demonstrates that the conditions from Lemma 42 and Lemma 44 are compatible to each other.

Corollary 49. (Reformulation of Corollary 43)

(i) Assume $k^{l}=k^{c}$ and $d_{1}^{c}=d_{1}^{l}=0$. For existence of $k \bar{l}_{2}$ in the biologically relevant range fulfilling $H_{2}\left(k \bar{l}_{2}\right)<0$, it is necessary and sufficient that $\hat{\alpha}>0, \tilde{\beta}<0, \hat{\alpha}(1-\bar{s})+\tilde{\delta} \bar{s} \geq 0$ and $\frac{-\tilde{\beta}^{2}}{4 \hat{\alpha} \bar{s}^{2}}+\tilde{\gamma}<0$. 
(ii) After possible renaming of variables, the condition $\hat{\alpha}>0$ is equivalent to $d_{2}^{c}>d_{2}^{l}$ and $p_{1}^{l} d_{2}^{l}>p_{1}^{c} d_{2}^{c}$. Consequently the following additional conditions are necessary and sufficient for existence of a biologically relevant $k \bar{l}_{2}$ with $H_{2}\left(k \bar{l}_{2}\right)<0$ :

- $\bar{s}<\frac{\hat{\alpha}}{\hat{\alpha}+\tilde{\delta}}, p_{1}^{c}>\frac{p_{1}^{l}\left(2 d_{2}^{l}-d_{2}^{c}\right)+\left(d_{2}^{c}\right)^{2}-d_{2}^{c} d_{2}^{l}}{d_{2}^{c}}, p_{1}^{l}>d_{2}^{c}\left(\Leftrightarrow \tilde{\beta}<0\right.$ while $p_{1}^{l} d_{2}^{l}>p_{1}^{c} d_{2}^{c}$ and $\left.\bar{s}>\frac{1}{2}\right)$,

- $\left.p_{1}^{c} \leq \frac{p_{1}^{l} d_{2}^{l}\left(d_{2}^{c}-d_{2}^{l}\right)(1-\bar{s})+d_{2}^{c} d_{2}^{l}\left(d_{2}^{c}-d_{2}^{l}\right) \bar{s}+p_{1}^{l}\left(d_{2}^{l}\right)^{2} \bar{s}}{d_{2}^{c}\left(d_{2}^{c}-d_{2}^{l}\right)(1-\bar{s})+\left(d_{2}^{c}\right)^{2} \bar{s}}(\Leftrightarrow \hat{\alpha}(1-\bar{s})+\tilde{\delta} \bar{s} \geq 0)\right)$,

- $\frac{-\tilde{\beta}^{2}}{4 \hat{\alpha} \bar{s}^{2}}+\tilde{\gamma}<0$.

Proof: The corollary follows from Propositions 27, 29, Lemmas 39, 40, 42, 44 and 45 and from Remarks 37 and 35.

We now elaborate on the constraints on $\bar{s}$.

Lemma 50. Assume $\hat{\alpha}(1-\bar{s})+\tilde{\delta} \bar{s} \geq 0$, then $\bar{s}<\frac{\hat{\alpha}}{\hat{\alpha}+\tilde{\delta}}$ is equivalent to

$$
p_{1}^{c}>\frac{d_{2}^{l}\left(p_{1}^{l} d_{2}^{c} \bar{s}-p_{1}^{l}\left(d_{2}^{c}-d_{2}^{l}\right)+\left(d_{2}^{c}\right)^{2} \bar{s}-d_{2}^{c} d_{2}^{l} \bar{s}\right)}{d_{2}^{c}\left(d_{2}^{c}(2 \bar{s}-1)+d_{2}^{l}(1-\bar{s})\right)} .
$$

\section{Proof:}

$$
\begin{aligned}
\bar{s} & <\frac{\hat{\alpha}}{\hat{\alpha}+\tilde{\delta}} \Leftrightarrow \\
\tilde{\delta} \bar{s} & <(1-\bar{s}) \hat{\alpha} \Leftrightarrow \\
p_{1}^{c} d_{2}^{c}\left(d_{2}^{c}(1-2 \bar{s})-d_{2}^{l}(1-\bar{s})\right) & <d_{2}^{l}\left(p_{1}^{l}\left(d_{2}^{c}-d_{2}^{l}\right)-p_{1}^{l} d_{2}^{c} \bar{s}-\left(d_{2}^{c}\right)^{2} \bar{s}+d_{2}^{c} d_{2}^{l} \bar{s}\right) \Leftrightarrow \\
p_{1} & >\frac{d_{2}^{l}\left(p_{1}^{l} d_{2}^{c} \bar{s}-p_{1}^{l}\left(d_{2}^{c}-d_{2}^{l}\right)+\left(d_{2}^{c}\right)^{2} \bar{s}-d_{2}^{c} d_{2}^{l} \bar{s}\right)}{d_{2}^{c}\left(d_{2}^{c}(2 \bar{s}-1)+d_{2}^{l}(1-\bar{s})\right)},
\end{aligned}
$$

since $p_{1}^{c} d_{2}^{c}\left(d_{2}^{c}(1-2 \bar{s})-d_{2}^{l}(1-\bar{s})\right)<0$.

Lemma 51. Denote:

- $P_{\text {max }}:=\frac{p_{1}^{l} d_{2}^{l}\left(d_{2}^{c}-d_{2}^{l}\right)(1-\bar{s})+d_{2}^{c} d_{2}^{l}\left(d_{2}^{c}-d_{2}^{l}\right) \bar{s}+p_{1}^{l}\left(d_{2}^{l}\right)^{2} \bar{s}}{d_{2}^{c}\left(d_{2}^{c}-d_{2}^{l}\right)(1-\bar{s})+\left(d_{2}^{c}\right)^{2} \bar{s}}$,

- $P_{\text {min }}:=\frac{d_{2}^{l}\left(p_{1}^{l} d_{2}^{c} \bar{s}+\left(d_{2}^{c}\right)^{2} \bar{s}-d_{2}^{c} d_{2}^{l} \bar{s}-p_{1}^{l} d_{2}^{c}+p_{1}^{l} d_{2}^{l}\right)}{d_{2}^{c}\left(2 d_{2}^{c} \bar{s}-d_{2}^{l} \bar{s}-d_{2}^{c}+d_{2}^{l}\right)}$,

- $P_{0}:=\frac{p_{1}^{l}\left(2 d_{2}^{l}-d_{2}^{c}\right)+\left(d_{2}^{c}\right)^{2}-d_{2}^{c} d_{2}^{l}}{d_{2}^{c}}$.

Assume $p_{1}^{l}>d_{2}$, then

(i) $P_{\max }>P_{\min }$,

(ii) $P_{\min }>P_{0}$. 
Proof: Since both denominators are positive (i) is equivalent to

$$
\begin{array}{rc}
\left(p_{1}^{l} d_{2}^{l}\left(d_{2}^{c}-d_{2}^{l}\right)(1-\bar{s})+d_{2}^{c} d_{2}^{l}\left(d_{2}^{c}-d_{2}^{l}\right) \bar{s}+p_{1}^{l}\left(d_{2}^{l}\right)^{2} \bar{s}\right)\left(2 d_{2}^{c} \bar{s}-d_{2}^{l} \bar{s}-d_{2}^{c}+d_{2}^{l}\right) & > \\
\left(d_{2}^{l}\left(p_{1}^{l} d_{2}^{c} \bar{s}+\left(d_{2}^{c}\right)^{2} \bar{s}-d_{2}^{c} d_{2}^{l} \bar{s}-p_{1}^{l} d_{2}^{c}+p_{1}^{l} d_{2}^{l}\right)\right)\left(\left(d_{2}^{c}-d_{2}^{l}\right)(1-\bar{s})+d_{2}^{c} \bar{s}\right) & \Leftrightarrow \\
\left(d_{2}^{c}-d_{2}^{l}\right)^{2}(1-\bar{s})\left(p_{1}^{l}-d_{2}^{c}\right)>0 . &
\end{array}
$$

Since both denominators are positive (ii) is equivalent to

$$
\begin{array}{rc}
d_{2}^{l}\left(p_{1}^{l} d_{2}^{c} \bar{s}+\left(d_{2}^{c}\right)^{2} \bar{s}-d_{2}^{c} d_{2}^{l} \bar{s}-p_{1}^{l} d_{2}^{c}+p_{1}^{l} d_{2}^{l}\right) & > \\
\left(p_{1}^{l}\left(2 d_{2}^{l}-d_{2}^{c}\right)+\left(d_{2}^{c}\right)^{2}-d_{2}^{c} d_{2}^{l}\right)\left(2 d_{2}^{c} \bar{s}-d_{2}^{l} \bar{s}-d_{2}^{c}+d_{2}^{l}\right) & \Leftrightarrow \\
\left(d_{2}^{c}-d_{2}^{l}\right)^{2}(2 \bar{s}-1)\left(p_{1}^{l}-d_{2}^{c}\right)>0 . &
\end{array}
$$

Remark 52. Due to Lemma 51 we obtain a stronger condition on $p_{1}^{c}$ :

$$
p_{1}^{c} \in\left(P_{\min }, P_{\max }\right] .
$$

Proposition 31 follows from:

Proposition 53. (Reformulation of Corollary 49)

(i) Assume $k^{l}=k^{c}$ and $d_{1}^{c}=d_{1}^{l}=0$. Then it is necessary and sufficient that $\hat{\alpha}>0, \tilde{\beta}<0$, $\hat{\alpha}(1-\bar{s})+\tilde{\delta} \bar{s} \geq 0$ and $\frac{-\tilde{\beta}^{2}}{4 \hat{\alpha} \bar{s}^{2}}+\tilde{\gamma}<0$ to obtain $H_{2}<0$ within the biologically relevant range of parameters.

(ii) After possible renaming of variables, the condition $\hat{\alpha}>0$ is equivalent to $d_{2}>d_{2}^{l}$ and $p_{1}^{l} d_{2}^{l}>p_{1} d_{2}$. Consequently, the following additional conditions are necessary and sufficient for existence of a biologically relevant $k \bar{l}_{2}$ with $H_{2}\left(k \bar{l}_{2}\right)<0$ :

- $p_{1}^{c}>P_{\text {min }}, p_{1}^{l}>d_{2}^{c}\left(\Leftrightarrow \tilde{\beta}<0\right.$ while $p_{1}^{l} d_{2}^{l}>p_{1}^{c} d_{2}^{c}$ and $\left.\bar{s}>\frac{1}{2}\right)$,

- $p_{1}^{c} \leq P_{\max }(\Leftrightarrow \hat{\alpha}(1-\bar{s})+\tilde{\delta} \bar{s} \geq 0)$,

- $\frac{-\tilde{\beta}^{2}}{4 \hat{\alpha} \bar{s}^{2}}+\tilde{\gamma}<0$.

(iii) $-\tilde{\beta}^{2}+4 \hat{\alpha} \tilde{\gamma} \bar{s}^{2}$ is a polynomial of order two in $p_{1}$, with a negative maximum order coefficient. Denote by $\tilde{p}_{\min }$ and $\tilde{p}_{\max }$ the zeros of this polynomial, if they are real. Then the conditions in (ii) are equivalent to

- $p_{1}^{l}>d_{2}^{c}$

- $p_{1}^{c} \in\left(P_{\min }, P_{\max }\right] \backslash\left[\tilde{p}_{\min }, \tilde{p}_{\max }\right]$.

If the zeros are not real, the only constraint on $p_{1}^{c}$ is $p_{1}^{c} \in\left(P_{\min }, P_{\max }\right]$. 
(iv) If the necessary and sufficient conditions are fulfilled, then the biologically relevant range of $k \bar{l}_{2}$ with $\mathrm{H}_{2}\left(k \bar{l}_{2}\right)<0$ is given by

$$
\left\{\left[0, \frac{1}{\bar{s}}-1\right] \cap\left(\frac{-\tilde{\beta}}{2 \hat{\alpha} \bar{s}^{2}}-\sqrt{\frac{\tilde{\beta}^{2}-4 \hat{\alpha} \bar{s}^{2} \tilde{\gamma}}{\left(2 \hat{\alpha} \bar{s}^{2}\right)^{2}}}, \frac{-\tilde{\beta}}{2 \hat{\alpha} \bar{s}^{2}}+\sqrt{\frac{\tilde{\beta}^{2}-4 \hat{\alpha} \bar{s}^{2} \tilde{\gamma}}{\left(2 \hat{\alpha} \bar{s}^{2}\right)^{2}}}\right)\right\} \neq \emptyset .
$$

Proof: (i) and (ii) follows from Propositions 27, 29, Lemmas 39, 40, 42, 44, 45, 50, 51 and from Remarks 37 and 35.

(iii) The maximum order coefficient is

$$
-\left(\bar{s}(1-\bar{s}) d_{2}^{c}\left(d_{2}^{c}-d_{2}^{l}\right)-\bar{s}^{2}\left(d_{2}^{c}\right)^{2}\right)^{2}-4\left(d_{2}^{c}\right)^{3}\left(d_{2}^{c}-d_{2}^{l}\right) \bar{s}^{4}\left(\frac{1}{\bar{s}}-1\right)<0
$$

therefore the positive part lies between the two zeros, if they are real. If quadratic polynomial with negative highest order term has no real zeros, then it is everywhere negative.

(iv) As in Lemma 25 we write $H_{2}(X)=\hat{\alpha} \bar{s}^{2} X^{2}+\tilde{\beta} X+\tilde{\gamma}$, with $\hat{\alpha}>0, \tilde{\gamma}>0$. Due to the assumptions it holds $\frac{-\tilde{\beta}^{2}}{4 \hat{\alpha} \bar{s}^{2}}+\tilde{\gamma}<0$, therefore the square root is real. Due to Lemma $39 \frac{-\tilde{\beta}}{2 \hat{\alpha} \bar{s}^{2}} \in$ $\left[0, \frac{1}{\bar{s}}-1\right]$, therefore the intersection is not empty.

Remark 54. The conditions originating from Proposition 53 by renaming $p_{i}^{c} \leftrightarrow p_{i}^{l}$, $a_{i}^{c} \leftrightarrow a_{i}^{l}$, $c_{i} \leftrightarrow l_{i}^{l}, d_{2}^{l} \leftrightarrow d_{2}^{c}$, are also necessary and sufficient.

Remark 55. The set of parameters characterized by Proposition 53 is not empty, e.g. set $a_{1}^{c}=$ $a_{1}^{l}=0.98039, p_{1}^{c}=22, p_{1}^{l}=4000, d_{1}^{c}=d_{1}^{l}=0, d_{2}^{c}=0.5, d_{2}^{l}=0.2, k^{l}=k^{c}=12.8 \cdot 10^{-10}$, $\bar{l}_{2}=0.15 / k^{l}$, which leads to $H_{2} \approx-0.061$.

Remark 34 (i) follows from $\frac{-\tilde{\beta}}{2 \hat{\alpha} \bar{s}^{2}}-\sqrt{\left(\frac{-\tilde{\beta}}{2 \hat{\alpha} \bar{s}^{2}}\right)^{2}-\frac{\tilde{\gamma}}{\hat{\tilde{\alpha}} \bar{s}^{2}}}>0$. 